Pacific

Journal of

Mathematics

FUSS-CATALAN ALGEBRAS AND CHAINS OF INTERMEDIATE SUBFACTORS

\title{
ZePh A. LANDAU
}

Volume 197 No. 2

February 2001 


\title{
FUSS-CATALAN ALGEBRAS AND CHAINS OF INTERMEDIATE SUBFACTORS
}

\author{
ZePh A. LANDAU
}

\begin{abstract}
We give a complete description of the generalized FussCatalan algebras: colored generalizations of the TemperleyLieb algebras, introduced by D. Bisch and V. Jones. For these chains of finite dimensional algebras, we describe a basis in terms of generators, and give a complete description, including the dimensions, of the irreducible representations.

We then consider an arbitrary subfactor containing a chain of intermediate subfactors. The higher relative commutants of a subfactor are an important tool for classifying the subfactor. We show the Fuss-Catalan algebras to be generically contained inside the higher relative commutants of the subfactor. Thus the Fuss-Catalan algebras provide an underlying structure for the higher relative commutants of any subfactor that contains a chain of intermediate subfactors.
\end{abstract}

\section{Introduction.}

Given an inclusion of type $\mathrm{II}_{1}$ factors $N \subset M$ of finite index, the basic construction yields a tower of $\mathrm{II}_{1}$ factors [Jo1]:

$$
N \subset M \subset M_{1} \subset M_{2} \subset \ldots
$$

along with a sequence of projections $e_{1}, e_{2}, e_{3}, \ldots$ with $M_{i}=\left\{M_{i-1}, e_{i}\right\}^{\prime \prime}$ (and $M_{1}=\left\{M, e_{1}\right\}^{\prime \prime}$ ). Intersecting this tower with the algebras $N^{\prime}$ and $M^{\prime}$ gives a double sequence of finite-dimensional algebras:

$$
\begin{aligned}
& N^{\prime} \cap N \quad \subset \quad N^{\prime} \cap M \quad \subset \quad N^{\prime} \cap M_{1} \subset \ldots \\
& M^{\prime} \cap M \quad \subset \quad M^{\prime} \cap M_{1} \subset \ldots
\end{aligned}
$$

known as the (opposite) standard invariant (see for example [Po1]). Much of the structure of $N \subset M$ (in some cases all of it [Po2]) is captured by the standard invariant, and therefore a full description of it is very desirable. It was pointed out in [Jo1] that $\left\{e_{1}, e_{2}, \ldots e_{i}\right\}^{\prime \prime} \subset N^{\prime} \cap M_{i}$ and $\left\{e_{2}, e_{3} \ldots e_{i}\right\}^{\prime \prime} \subset M^{\prime} \cap M_{i}$ for any subfactor $N \subset M$ and thus a description of the algebras $\left\{1, e_{1}, e_{2}, \ldots e_{i}\right\}^{\prime \prime}$ provides an underlying structure for all standard invariants. These algebras, the Temperley-Lieb algebras, were fully described in this same paper [Jo1], giving the structure of the reduced 
words, the Brattelli diagram for the inclusions, as well as the value of the trace.

D. Bisch and V. Jones analyzed the corresponding situation when a subfactor $N \subset M$ is known to have an intermediate subfactor $N \subset P \subset M$ [BiJo1]. Here, the presence of $P$ leads to added structure (i.e., additional projections) in the standard invariant. This added structure was analyzed and shown to be strongly related (in the generic case isomorphic) to a "colored" string algebra termed the Fuss-Catalan algebra $F C_{k, n}$. A full analysis of these algebras was given, with the exception of describing a basis set. In addition, some of the work in [BiJo1] addressed the more general situation of a subfactor $N \subset M$ with a chain of $k$ intermediate subfactors:

$$
N \subset P^{1} \subset P^{2} \subset \ldots P^{k} \subset M
$$

and it was stated that the main results can be generalized to this situation.

The following is this generalization. We analyze the structure imposed on the standard invariant by a chain of $k$ intermediate subfactors. Three algebras are discussed:

1) An abstract algebra $A A_{k, n}$ that is defined by generators and relations,

2) The colored string algebras $F C_{k, n}$ introduced in [BiJo1],

3) The algebras $I A_{k, n}$ that are the concrete algebras generated by projections corresponding to the intermediate subfactors $P^{1}, P^{2}, \ldots P^{k}$.

Sections 2-5 define the algebras $A A_{k, n}$ and analyze their structure. Section 3 gives a spanning set of words. Sections 4 and 5 count these words by giving a correspondence with certain types of labeled planar trees and then counting these trees. Section 6 defines the algebra $F C_{k, n}$, giving a generating set. Section 7 puts this work together to establish the isomorphism between $A A_{k, n}$ and $F C_{k, n}$, and in the process establishes that the spanning set given for $A A_{k, n}$ is actually a basis. Section 8 mimics the elegant structural analysis for $F C_{1, n}$ done in [BiJo1], giving an inductive description of the Bratteli diagram for

$$
F C_{k, 0} \subset F C_{k, 1} \subset F C_{k, 2} \ldots
$$

Section 9 develops explicit formulas for the dimensions of the irreducible representations of $F C_{k, n}$. Section 10 again mimics work in [BiJo1], giving a description of the trace on $F C_{k, n}$. Section 11 establishes the connection with subfactors: defining $I A_{k, n}$ and establishing the isomorphism with $A A_{k, n}$ (and thus with $F C_{k, n}$ ) generically. In addition Section 11 addresses the non-generic case, showing $I A_{k, n}$ to be a quotient of $A A_{k, n}$.

It is worth pointing out the similarities and differences between what is contained in [BiJo1] and what is contained here. The work in [BiJo1], though mainly concerned with the $k=1$ case, provided a very nice road map for what appears here. The broad structure of the argument in both papers is the same: defining $A A_{k, n}$ and $F C_{k, n}$, proving them isomorphic, 
establishing the isomorphism (homomorphism in the non-generic case) to $I A_{k, n}$, and finally analyzing the structure of $F C_{k, n}$ (and hence the structure of $\left.I A_{k, n}\right)$. Sections 7, 8, 10, and 11 consist of arguments from [BiJo1] adapted to the general case. In addition, many details particular to the analysis of $F C_{k, n}$ (Section 8), and the analysis of the trace (Section 10), though presented in [BiJo1] only for $k=1$, carry through merely by replacing statements with 2 variables to statements with $k$ variables. These restatements are referenced to [BiJo1] and given without proofs.

What is completely new in this work falls into two main categories: the description of a basis for the algebras $A A_{k, n}$ (Sections 2 - 6) and the computation of the dimensions of the irreducible representations of $F C_{k, n}$ (Section 9 ) as well as the description of the Bratteli diagram for the algebras $F C_{k, n}$ (Theorem 10). The use of the basis for $A A_{k, n}$ establishes $A A_{k, n} \cong F C_{k, n}$ which had been conjectured in [BiJo1], and also significantly simplifies and clarifies the proof of the case $A A_{1, n} \cong F C_{1, n}$ [BiJo1]. Explicit formulas for the dimensions of the irreducible representations of $F C_{k, n}$ are given using combinatorial arguments. (This computation for $k=1$ was done by other means in $[\mathbf{B i J o 2}]$.

Finally, because most of the material in Sections 1-10 does not involve subfactors, it is accessible to non-specialists. Moreover the combinatorial aspects of Sections 3 - 5 and 9 are of independent interest.

\section{The Algebra $A A_{k, n}$.}

We begin by defining an abstract algebra $A A_{k, n}\left(\alpha_{0}, \alpha_{1}, \ldots \alpha_{k}\right)$ (this same algebra is considered independently in $[\mathbf{D F r}]$ ).

Definition 1. Let $A A_{k, n}\left(\alpha_{0}, \alpha_{1}, \ldots \alpha_{k}\right), \alpha_{i} \in \mathbb{C}$ be the abstract algebra generated by:

$$
\left\{1,{ }_{a} E_{i}\right\} \quad 1 \leq i \leq n-1, \quad 0 \leq a \leq k,
$$

subject to the relations:

0) $1{ }_{a} E_{i}={ }_{a} E_{i} 1={ }_{a} E_{i}$

1) ${ }_{a} E_{i b} E_{i}=\max (a, b) E_{i}$

2) ${ }_{a} E_{i b} E_{j}={ }_{b} E_{j a} E_{i}$

for $|i-j| \geq 2$ or

$|i-j|=1$ and

$a+b<k$.

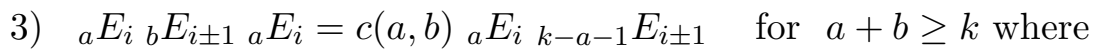
we set ${ }_{-1} E_{i \pm 1}=1$. 
Here $c(a, b)=\frac{k-a-1 c_{i+1 k-b-1} c_{i}}{{ }_{a} c_{i} c_{i+1}}$, with
\[ c_{a} c_{i}=\left\{\begin{array}{ll}\alpha_{0} \alpha_{1} \cdots \alpha_{a} & \text { for } i \text { even, } \\ \alpha_{k} \cdots \alpha_{k-a} & \text { for } i \text { odd, }\end{array} \text { and }{ }_{-1} c_{i}=1 .\right. \]

\section{Remarks.}

1) Relations 1) and 2) imply that ${ }_{a} E_{k b} E_{k \pm 1 a} E_{k}={ }_{a} E_{k b} E_{k \pm 1}$ for $a+b<k$.

2) Relations 1) and 3) imply ${ }_{a} E_{k b} E_{k \pm 1 d} E_{k}$ can always be reduced to a word with two or fewer terms.

\section{Abacus Construction.}

Abstractly these relations are slightly daunting, but there is a nice visualization of the words of this algebra. Consider an abacus with $n-1$ horizontal bars labeled from bottom to top by the integers 1 through $n-1$. In addition, have the bars of the abacus spaced $k$ units apart. Now represent ${ }_{a} E_{j}$ by a bead of radius $a$ units, hanging on the $j$ th horizontal bar. Thus the word

$$
{ }_{1} E_{32} E_{21} E_{14} E_{30} E_{2} \in A A_{4,5}\left(\alpha_{0}, \ldots \alpha_{4}\right)
$$

would be depicted by the following picture:

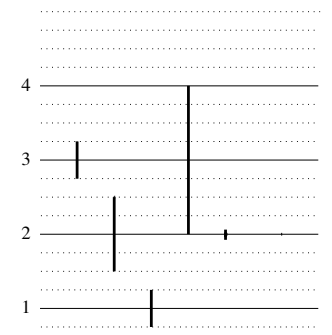

With this description, relation 1) has the following visual interpretation:

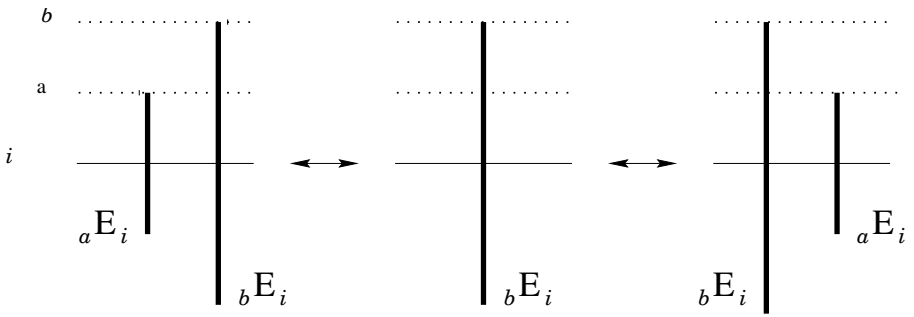

(assuming $a<b$ ).

Relation 2) is given by: 


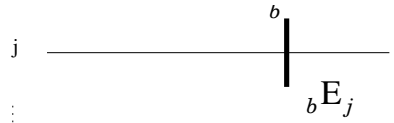

$i+1$

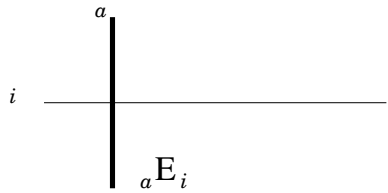

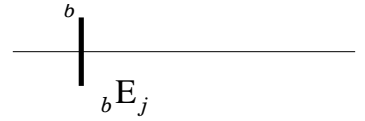

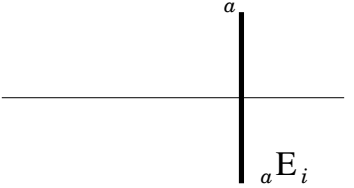

$($ for $|i-j| \geq 2)$

and
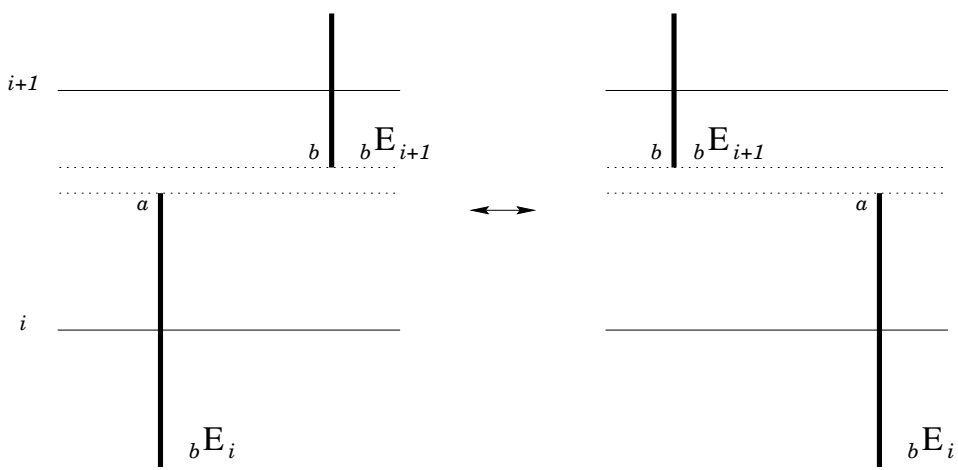

(for $|i-j|=1$ and $a+b<k$ )

Note. The abacus picture was motivated by interpreting this second relation. We allow two generators to commute (slide by each other) if there is space between their respective beads. We note that if there is no room (i.e., $a+b=k$ ), we do not allow them to slide by each other (commute). (If you like, imagine that the abacus is not that well made so that the beads are just a little larger than they are supposed to be.) Finally, relation 3) is visually interpreted as:

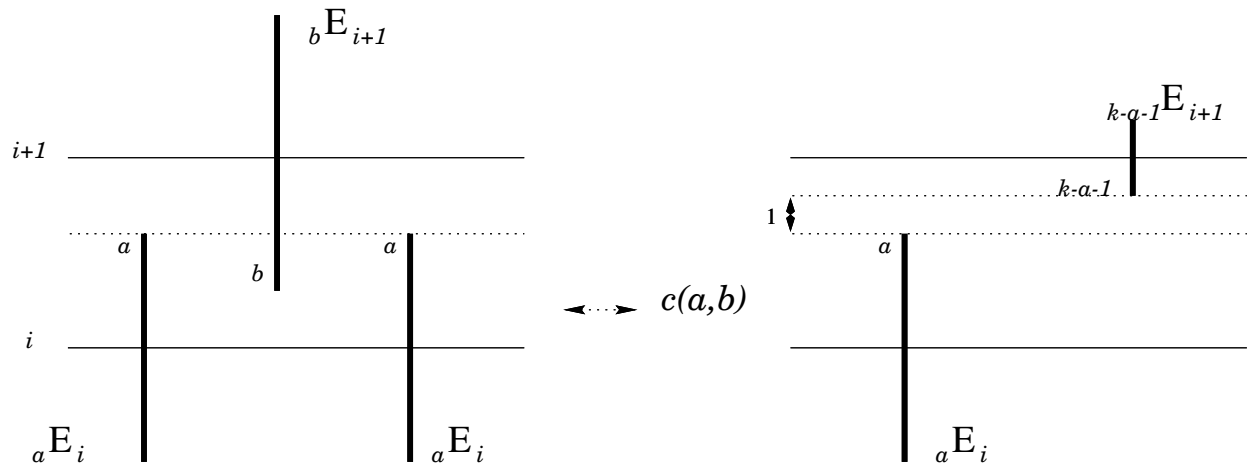


The abacus picture is very helpful for what follows, and the reader is encouraged to think of manipulating words in $A A_{k, n}\left(\alpha_{0}, \cdots a_{k}\right)$ via these pictures.

We now analyze the structure of $A A_{k, n}$. In the next section, we will show that a certain subset of $A A_{k, n}$, defined to be the set of words of reduced form, linearly spans $A A_{k, n}$. In Sections 4 and 5 we will count the number of these reduced words. Later, in Section 7 we will show that the number of these words is equal to the dimension of $A A_{k, n}$ and that therefore the words of reduced form are a basis of $A A_{k, n}$.

\section{A Spanning Set for $A A_{k, n}$.}

Define a function $s$ on the formal words in the $\left\{{ }_{a} E_{k}\right\}$ as follows:

$$
\text { for } \quad w=\prod_{i=1 a(i)}^{l} E_{b(i)}, \quad \text { define } \quad s(w)=\sum_{i=1}^{l}(a(i)+1) .
$$

Note that we are defining $s$ on the formal words, (i.e., the words in the free product of the generators), not on the elements of $A A_{k, n}$. We will call $s(w)$ the size of $w$.

Definition 2. A word $w$ in the free product of the generators will be called minimal if $s(v) \geq s(w)$ for any $v \sim w$. Here we define $v \sim w$ to mean that as elements of $A A_{k, n}\left(\alpha_{0}, \cdots \alpha_{k}\right), v=c w$ with $c$ a nonzero scalar.

We introduce the notation $r_{M(i), m(i)}$ to mean a word of the form

$$
r_{M(i), m(i)}=\prod_{j=M(i)}^{m(i)} a(i, j) E_{j} \quad \text { with } \quad m(i) \leq M(i), 0 \leq a(i, j) \leq k,
$$

where we take the product in decreasing order from $j=M(i)$ to $j=m(i)$. Pictorially $r_{M(i), m(i)}$ represents a word with its first bead on the $M(i)$ th level, and each subsequent bead on the next lower level, with the last bead on the $m(i)$ th level.

Lemma 1. For $m(i) \leq l \leq M(i)$, the words $r_{M(i), m(i)}{ }_{a} E_{l}$ and ${ }_{a} E_{l} r_{M(i), m(i)}$ are not minimal.

Proof. For $l \neq m(i)$,

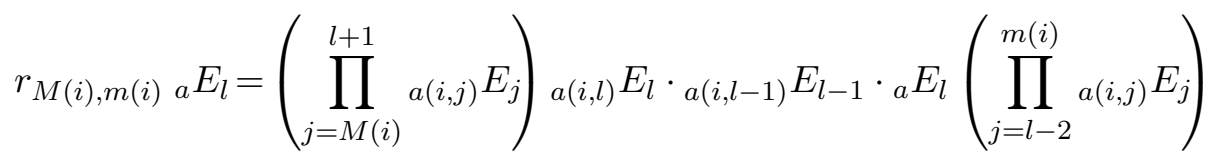

which is not minimal since ${ }_{a(i, l)} E_{l a(i, l-1)} E_{l-1 a} E_{l}$ is not minimal by the remark after Definition 1. The abacus picture that corresponds to the above calculation is as follows: 

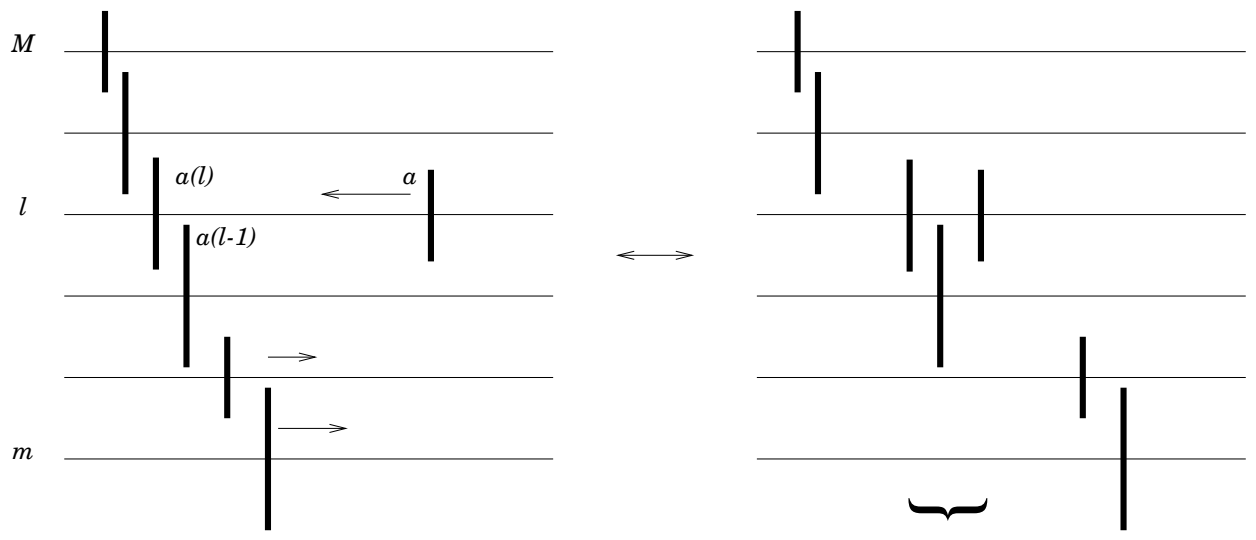

This reduces

For $k=m(i)$ we have

$$
r_{M(i), m(i)} \cdot{ }_{a} E_{m(i)}=\left(\prod_{j=M(i)}^{m(i)+1} a(i, j) E_{j}\right) \cdot{ }_{a(i, m(i))} E_{m(i) a} E_{m(i)}
$$

which reduces by relation 1 . By turning the pictures upside down we have a proof for ${ }_{a} E_{k} \cdot r_{M(i), m(i)}$.

Lemma 2. Given $v$ minimal, then $v \sim w$ with $s(v)=s(w)$ and $w$ of the form

$$
\begin{aligned}
& \qquad w=\prod_{i=1}^{l} r_{M(i), m(i)}, \quad \text { with } \\
& M(1)<M(2)<M(3) \ldots, \\
& m(1)<m(2)<m(3) \ldots
\end{aligned}
$$

Proof. Given $v=\prod_{i=1 a(i)}^{l} E_{b(i)}$, consider the related word $\hat{v}$ in the Temperley-Lieb algebra: $\hat{v}=\prod_{i=1}^{l} e_{b(i)}[\mathbf{J} \mathbf{0 1}]$. Now reduce $\hat{v}$ using the TemperleyLieb relations:

1') $e_{i}^{2}=e_{i}$,

2') $e_{i} e_{j}=e_{j} e_{i}, \quad|i-j| \geq 2$,

3') $e_{i} e_{i \pm 1} e_{i}=\lambda e_{i}$.

Any use of 2') can be mirrored by the use of relation 2) on $v$. If the reduction uses $1^{\prime}$ ) or $3^{\prime}$ ) on $\hat{v}$, the corresponding use of relations 1 ), 3 ) or the remarks immediately following the definition of $A A_{k, n}$ will reduce the size of $v$, violating the minimality of $v$. Thus, using only relation 2', $\hat{v}$ can be put in the reduced form described in [Jo1, p. 14]. Mirroring these moves on $v$ gives us a word of the form described in the statement of the lemma. 
We give the following two definitions. For now we are interested in the definition of a reduced block. The definition of an $n$-block will facilitate a later discussion.

In what follows, $l$ is an integer, $m$ and $M$ are maps from $\{1,2, \ldots, l\}$ to the integers and $a$ is a map from $A=\{(i, j): 1 \leq i \leq l, m(i) \leq j \leq M(i)\}$ to $\{0,1, \ldots, k\}$.

Definition 3. A word $w$ in the generators of $A A_{k, n}$ is called a block, and denoted by $(m, M, l, a)$, if it is of the form:

$$
w=\prod_{i=1}^{l} r_{M(i), m(i)} \quad \text { with }
$$

b1. $\quad M(1)<M(2)<M(3) \cdots<M(l)$,

$m(1)<m(2)<m(3) \cdots<m(l)$, $m(i+1) \leq M(i)$.

b2. $a(i, j)=a(i-1, j-2)$ whenever $a(i, j), a(i, j-1), a(i-1, j-1), a(i-$ $1, j-2)$ are all defined (i.e., $(i, j),(i, j-1),(i-1, j-1),(i-1, j-1) \in A)$.

A block with $m(1)=1$ and $M(l)=n$ is called an $n$-block.

Definition 4. A word $w$ in the generators of $A A_{k, n}$ is called a reduced block, and denoted by $(m, M, l, a)$ if it is a block and satisfies the additional condition:

$$
\begin{array}{ll}
\text { b3. } & a(i, j)+a(i, j-1) \geq k, \\
& a(i, j)+a(i+1, j+1) \geq k .
\end{array}
$$

\section{Remark.}

We present a brief visual description of the reduced blocks in the abacus picture. A reduced block is a sequence of descending strings of beads (each descending string being an $r_{M(i), m(i)}$ with beads starting at the $M(i)$ th level and ending at the $m(i)$ th level). Condition b1 tells us that the next descending string starts (resp. ends) at a higher level than the previous one starts (resp. ends). In addition, the ending of the next descending string cannot be higher than the start of the previous one. Condition b2 requires that if we ever see a diamond shape of beads as follows: 


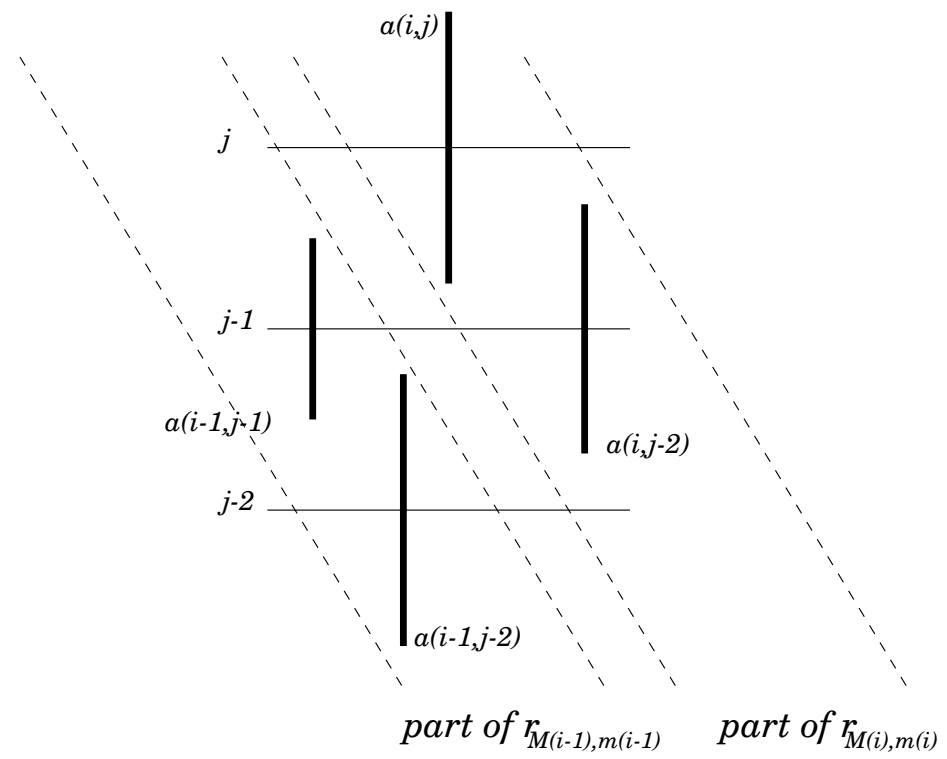

the top $(a(i, j))$ and bottom $(a(i-1, j-2))$ bead must have the same length. Condition b3 tells us that any bead at level $j$ will be large enough to touch the closest beads to it that hang at the $j \pm 1$ level.

We now describe how to write a word in $A A_{k, n}$ as a product of reduced blocks. Suppose we have $w=\prod_{i=1}^{l} r_{M(i), m(i)}$ minimal, of the form of Lemma 2 , and with the added requirement that $l$ is maximal. Let $L=\{i: m(i+1)>$ $M(i)\}$. Label the elements of $L$ in increasing order $l_{1}, l_{2}, \ldots, l_{d}$, so that $1 \leq l_{1}<l_{2} \ldots l_{d}<l$. Define

$$
\begin{aligned}
w_{1} & =\prod_{i=1}^{l_{1}} r_{M(i), m(i)}, & \\
w_{i} & =\prod_{i=l_{i-1}+1}^{l_{i}} r_{M(i), m(i)}, & \text { for } 2 \leq i \leq d, \\
w_{d+1} & =\prod_{i=l_{d}+1}^{l} r_{M(i), m(i)} . &
\end{aligned}
$$

Thus we have

$$
w=\prod_{m=1}^{d+1} w_{m} .
$$

Lemma 3. The $w_{m}$ as defined above are reduced blocks.

Proof. The $w_{m}$ clearly satisfy conditions b1: the first two because $w$ does, and the last due to the choice of the $l_{m}$. To show b2 we assume the existence 
of $a(i, j), a(i, j-1), a(i-1, j-1), a(i-1, j-2)$. We can use the commuting relations to write:

$$
w_{i}=\cdots_{a(i-1, j-1)} E_{j-1 \quad a(i-1, j-2)} E_{j-2 \quad a(i, j)} E_{j a(i, j-1)} E_{j-1} \cdots
$$

But this word is not of minimal size unless $a(i-1, j-2)=a(i, j)$. We demonstrate this with an example in $A A_{4, n}\left(\alpha_{0}, \alpha_{1} \ldots \alpha_{4}\right)$ along with its abacus interpretation.

$$
\begin{gathered}
{ }_{4} E_{23} E_{11} E_{33} E_{2} \rightarrow\left({ }_{4} E_{22} E_{2}\right){ }_{3} E_{11} E_{3}\left({ }_{2} E_{23} E_{2}\right) \\
\rightarrow{ }_{4} E_{2}\left({ }_{2} E_{23} E_{12} E_{2}\right){ }_{1} E_{33} E_{2} \rightarrow c_{3}(2,3){ }_{4} E_{2}\left({ }_{2} E_{21} E_{1}\right){ }_{2} E_{33} E_{2} \\
\rightarrow c_{3}(2,3){ }_{4} E_{21} E_{11} E_{33} E_{2}
\end{gathered}
$$
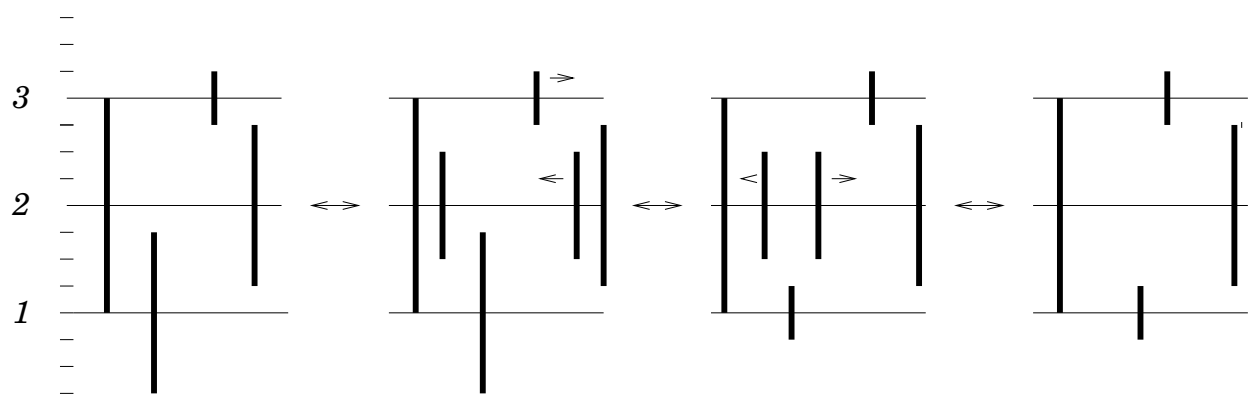

The same series of manipulations works on a general word of this form, though the notation becomes somewhat cumbersome.

Finally, for b3, suppose $a\left(i_{0}, j_{0}\right)+a\left(i_{0}, j_{0}-1\right)<k$. Thus ${ }_{a\left(i_{0}, j_{0}\right)} E_{j_{0}}$

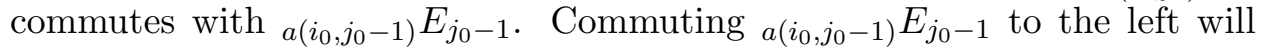
contradict minimality of $w$ (Lemma 1$)$ unless $M\left(i_{0}-1\right)<j_{0}-1$. Commuting $a\left(i_{0}, j_{0}\right) E_{j_{0}}$ to the right will yield a contradiction by the same lemma unless $m\left(i_{0}+1\right)>j_{0}$. In this case, let

$$
r_{1}=\prod_{j=M\left(i_{0}\right)}^{j_{0}} a\left(i_{0}, j\right) E_{j}, \quad r_{2}=\prod_{j=j_{0}-1}^{m\left(i_{0}\right)} a\left(i_{0}, j\right) E_{j},
$$

so that $r_{M\left(i_{0}\right), m\left(i_{0}\right)}=r_{1} r_{2}$. Note $r_{1}$ and $r_{2}$ commute because $a\left(i_{0}, j_{0}\right)+$ $a\left(i_{0}, j_{0}-1\right)<k$. Then we can rewrite

$$
\begin{gathered}
w=w_{1} w_{2} \ldots w_{m-1}\left(\prod_{j=l_{m-1}+1}^{i_{0}-1} r_{M(j), m(j)}\right) \\
\cdot r_{2} r_{1}\left(\prod_{j=i_{0}+1}^{l_{m}} r_{M(j), m(j)}\right) w_{m+1} w_{m+2} \ldots w_{d}
\end{gathered}
$$

which is of the desired form with one more term, thus contradicting the maximality of $l$. 
To clarify, we include the corresponding picture:

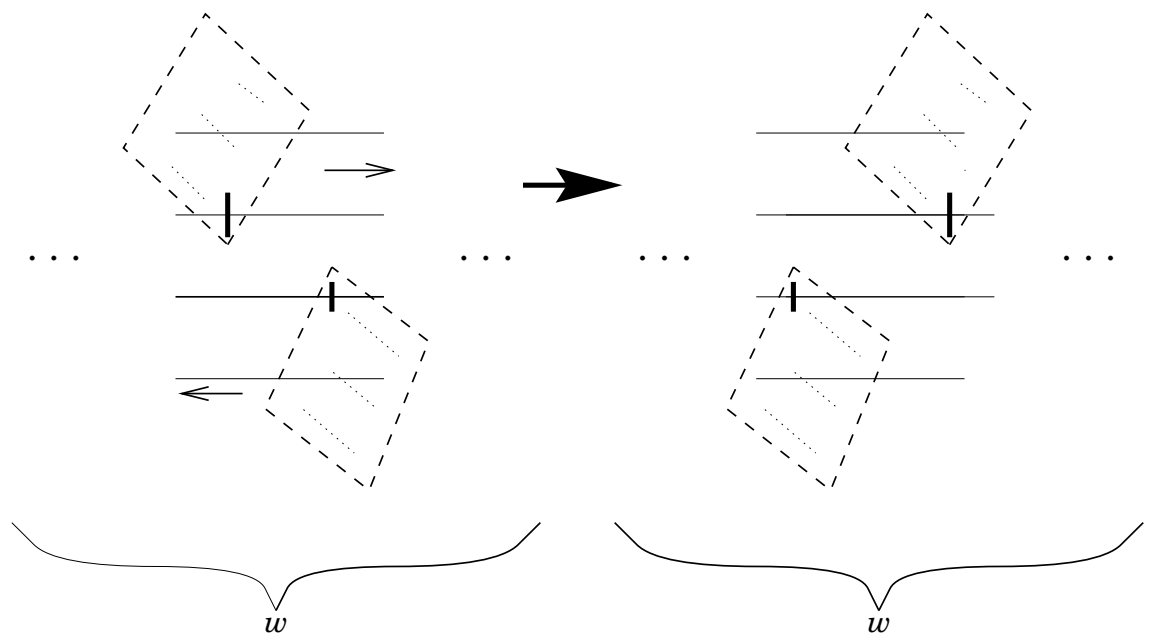

The two beads drawn are ${ }_{a\left(i_{0}, j_{0}+1\right)} E_{j_{0}+1}$ and ${ }_{a\left(i_{0}, j_{0}\right)} E_{j_{0}}$. The two dotted boxes are meant to surround those beads that represent $r_{1}$ and $r_{2}$. Because $M\left(i_{0}-1\right)<j_{0}-1$ and $m\left(i_{0}+1\right)>j_{0}$, the two boxes can slide by each other to give a configuration that contradicts the maximality of $l$, the number of $r$ 's in $w$.

A slightly quicker argument holds for the second part of condition b3. Suppose $a(i, j)+a(i+1, j+1)<k$, then ${ }_{a(i, j)} E_{j}$ and ${ }_{a(i+1, j+1)} E_{j+1}$ commute. Commuting ${ }_{a(i, j)} E_{j}$ to the right and using Lemma 1 we get a contradiction on the minimality of $w$ unless $m(i+1)=j+1$. Commuting ${ }_{a(i+1, j+1)} E_{j+1}$ to the left will again contradict minimality of $w$ unless $M(i)=j$. But this is impossible since $M(i)=j<m(i+1)$ means that ${ }_{a(i, j)} E_{j}$ and ${ }_{a(i+1, j+1)} E_{j+1}$ would be in different $w_{i}$.

We are now ready to describe what will turn out to be a basis for $A A_{k, n}$. Definition 5. A word $w$ will be called in reduced form if:

1) $w=1$,

or

2) $w=\prod_{i=1}^{d} w_{i}$ with a) the $w_{i}$ reduced blocks,

b) $\min \left(w_{i+1}\right)>\max \left(w_{i}\right)$.

where $\min (w)=\min \left\{j:{ }_{a} E_{j}\right.$ appears in $\left.w\right\}$, $\max (w)=\max \left\{j:{ }_{a} E_{j}\right.$ appears in $\left.w\right\}$.

Definition 6. Let $S_{k, n}$ to be the set of words of reduced form inside $A A_{k, n}$.

So far we have shown that any word can be put in the above reduced form (Lemma 3). Thus $S_{k, n}$ is a spanning set for $A A_{k, n}$ and $\operatorname{dim}\left(A A_{k, n}\right) \leq\left|S_{k, n}\right|$. Our next task is to count these words.

Our plan of attack for counting words of reduced form is as follows: 
1) Show a 1-1 correspondence between reduced blocks and certain pictures we call Kauffman (k,n)-diagrams.

2) Then establish a 1-1 correspondence between the Kauffman (k,n)diagrams and planar $(k, n)$-trees with labeled vertices.

3) Finally, count the number of planar $(k, n)$-trees using a recursion relation. Combine this with the relationship between reduced blocks and elements of $S_{k, n}$ to count the words of reduced form.

\section{Kauffman Diagrams and Planar Trees.}

In this section we give the 1-1 correspondences listed as items 1 and 2 in the above plan. First, we introduce the notion of a Kauffman diagram, an object defined in $[\mathbf{K a}]$ :

Definition 7. Let $D_{n}$ be a rectangle with $n$ marked points on the top of the boundary and $n$ marked points on the bottom. We define a Kauffman $n$-diagram to be a picture sitting inside $D_{n}$, consisting of $n$ non-intersecting curves that begin and end at distinct marked boundary points. We will consider two such diagrams equal if they are isotopically equivalent (keeping the boundary fixed).

We note that a Kauffman diagram is completely characterized by which marked boundary points are joined together. For simplicity we label the marked boundary points 1 through $2 n$ clockwise starting at the top left and ending at the bottom left. Then the Kauffman $n$-diagrams are in one-to-one correspondence with functions $J:[1,2 n] \rightarrow[1,2 n]$ with the following three properties:

1) $J(i) \neq i$,

2) $J^{2}=\mathrm{id}$,

3) If $i \leq j \leq J(i)$ then $i \leq J(j) \leq J(i)$.

We note that condition 3 characterizes the fact that the curves in a Kauffman diagram do not intersect (that is, the planarity of the Kauffman diagram). We will call a function $J$ with the three properties listed above a planar n-function.

We note that the curves in a Kauffman $n$-diagram divide $D_{n}$ into $n+1$ pieces. We will call a labeled Kauffman $(k, n)$-diagram a Kauffman diagram equipped with a labeling of each of its $n+1$ pieces by an integer in the interval $[0, k]$.

Lemma 4. With the exception of the identity Kauffman n-diagram: 


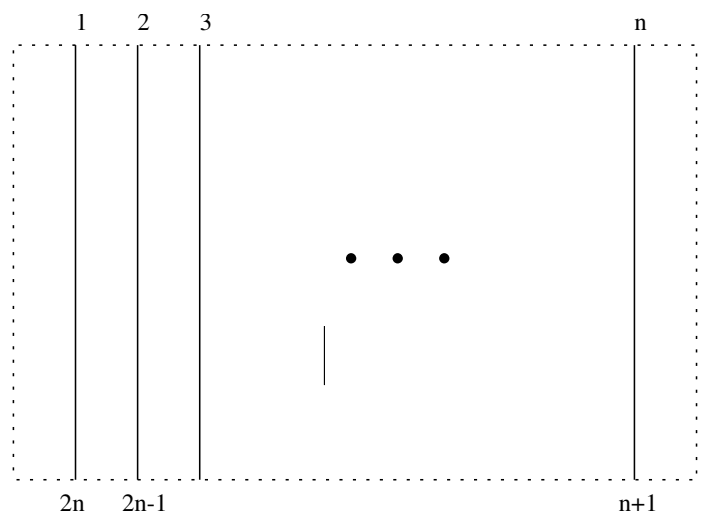

all Kauffman diagrams have at least one curve connecting adjacent points on the bottom of $D_{n}$ (i.e., there exists $i: n+1 \leq i<2 n$ with $J(i)=i+1$ ). Proof. For a given Kauffman diagram consider the smallest $l \geq n+1$ with $J(l) \neq 2 n+1-l$. (If no such $l$ exists then the diagram is the identity diagram.) The curve joining $l$ to $J(l)$ divides $D_{n}$ into two parts. Consider the part that includes the point $l+1$. By choice of $l$, unless $J(l)=l+1$ (in which case we are done), this part contains more points on the bottom row (i.e., with label $>l$ ) than points on the top row (i.e., with labels $<2 n+1-l$ ). Thus two points on the bottom must be joined together. Take a pair of points on the bottom that are joined together and that are minimal with respect to the number of points between them. By minimality, the points must be consecutive (since the planarity of Kauffman diagrams ensures that all the points between would be connected to each other).

Consider the following two pictures:
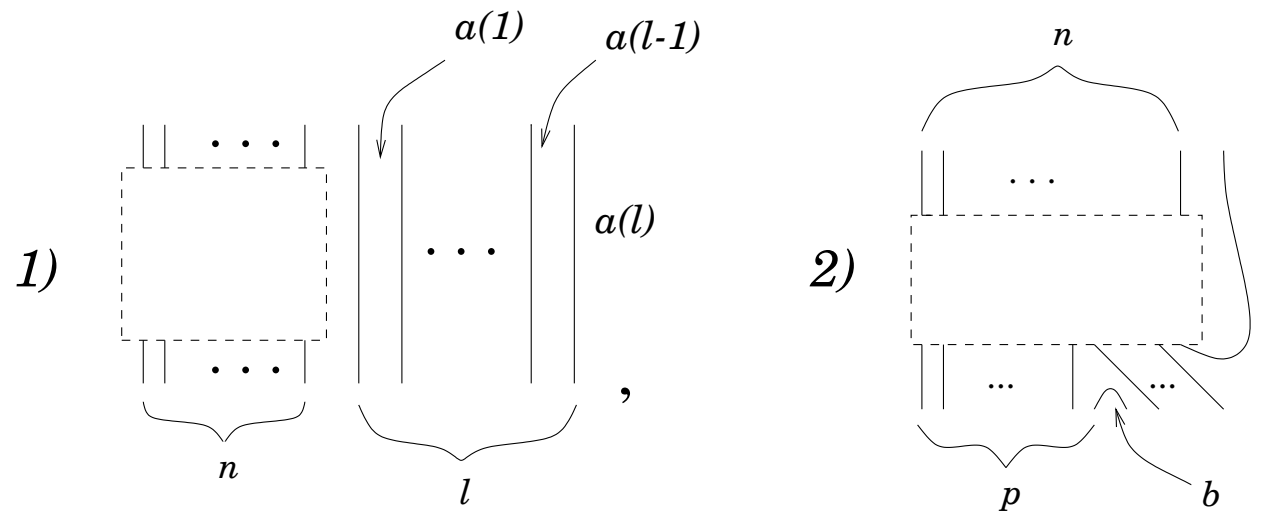

with $a(1), \ldots, a(l), b$ integers in the interval $[0, k]$. Both can be thought of as operators on labeled Kauffman $(k, n)$-diagrams. For a given labeled Kauffman $(k, n)$-diagram $K$, place $K$ inside the dotted rectangle aligning the marked boundary points with the strands leaving the rectangle. The resulting bigger rectangle is a labeled Kauffman $(k, n+l)$-diagram in the 
first case and a labeled Kauffman $(k, n+1)$-diagram in the second case. We will call the first operator $I_{l, n}(a(1), \ldots, a(l))$ and the second $\theta_{p, n}(b)$. We provide an example for clarification:

Given

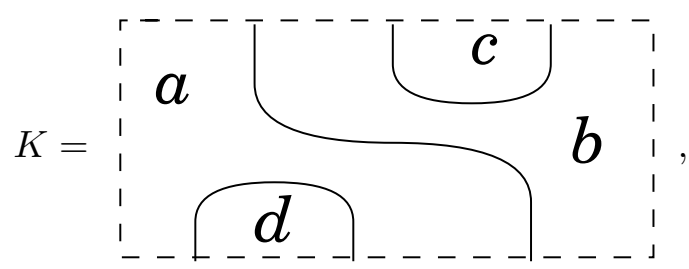

we have

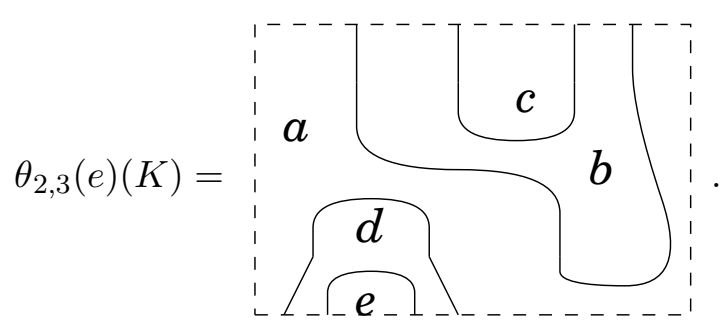

We now establish a 1-1 correspondence between $\operatorname{Kauffman}(k, n)$-diagrams and $n+1$ - blocks (recall Definition 3). Let $\psi:\{$ Kauffman $n$-diagrams $\} \rightarrow$ $\{n+1$ - blocks $\}$ be inductively defined as follows:

Given a labeled Kauffman $(k, n)$-diagram $K$,

$1)$ If $K$ is the Kauffman $(k, n)$-diagram:

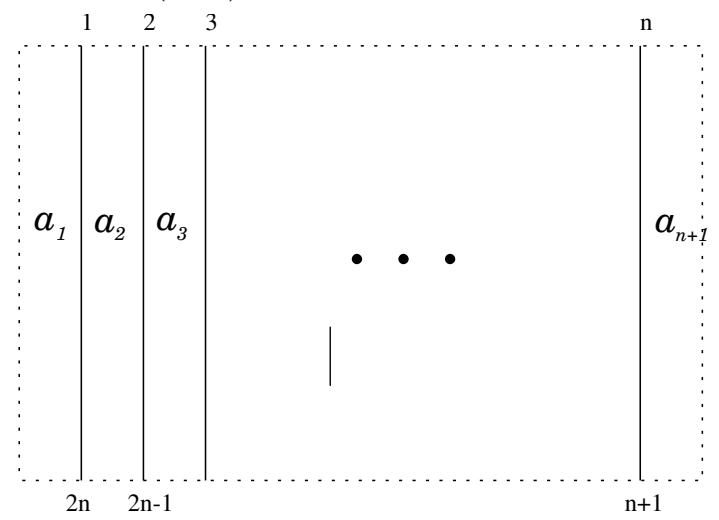

then $\psi(K)=(m, M, l, a)$ with $l=1, m(1)=1, M(1)=n+1, a(1, i)=$ $a_{i}$.

2) Otherwise choose $j$ maximal so that $K$ is in the image of $I_{j, n-j}(*)$ (i.e., $j$ is the number of vertical strands at the right of $K$ ). Write $K=I_{j, n-j}(a(1), \ldots, a(j))\left(K^{\prime}\right)$ with labels $a(1), \ldots, a(j)$ and Kauffman $(k, n-j)$-diagram $K^{\prime}$ uniquely determined from $K$ and $j$.

3) By Lemma $4, K^{\prime}$ has two consecutive bottom boundary points that are connected and thus $K^{\prime}$ is in the image of $\theta_{p, n-j-1}(*)$ for at least 
one $p$. Choose $p$ maximal and write $K^{\prime}=\theta_{p, n-j-1}(b)(L)$ with label $b$ and Kauffman (k,n-j-1)-diagram $L$ uniquely determined from $K^{\prime}$ and $p$.

4) Set $\psi(L)=\left(m^{\prime}, M^{\prime}, l^{\prime}, a^{\prime}\right)$. Define $\psi(K)=(m, M, l, a)$ inductively as:

- $l=l^{\prime}+1$

- $m(i)=m^{\prime}(i), M(i)=M^{\prime}(i)$ for $1 \leq i \leq l^{\prime}$

- $m(l)=p, M(l)=\mathrm{n}$

- $a\left(i^{\prime}, j^{\prime}\right)=a^{\prime}\left(i^{\prime}, j^{\prime}\right)$ for $1 \leq i^{\prime} \leq l^{\prime}$

- $a\left(l, j^{\prime}\right)=a\left(l-1, j^{\prime}-2\right)$ for $p<j^{\prime} \leq M^{\prime}(l)+1$

- $a(l, p)=b$

- $a\left(l, M^{\prime}(l)+1+i\right)=a(i)$ for $1 \leq i \leq j$.

We define what will turn out to be the inverse of $\psi$. Let the map

$$
T:\{n+1 \text {-blocks }\} \rightarrow\{\text { Kauffman }(k, n) \text {-diagrams }\}
$$

be inductively defined as follows:

Given an $n+1$-block $(m, M, l, a)$,

1) If $l=1$ then set

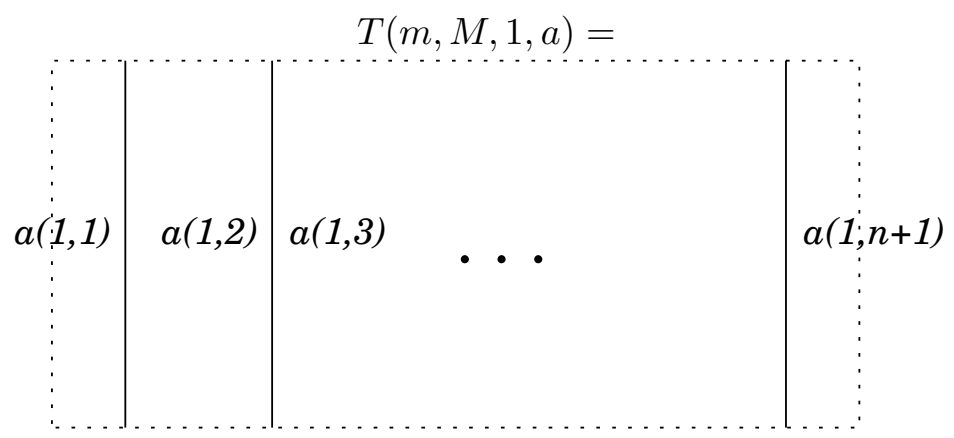

2) Otherwise, let

$$
\begin{gathered}
j=M(l)-M(l-1)-1, \\
\bar{a}=(a(l, M(l-1)+2), a(l, M(l-1)+3), \ldots, a(l, M(l-1)+j+1))
\end{gathered}
$$

and define

$$
T(m, M, l, a)=I_{j, M(l-1)}(\bar{a})\left(\theta_{m(l), n-j-1}\left(T\left(m^{\prime}, M^{\prime}, l-1, a^{\prime}\right)\right)\right)
$$

where the functions $m^{\prime}(i)$ and $M^{\prime}(i)$ are the functions $m(i)$ and $M(i)$ restricted to the domain $\{i: 1 \leq i \leq l-1\}$ and $a^{\prime}$ is just $a$ restricted to the part of the domain of $a$ with the first coordinate between 1 and $l-1$. We include the visual interpretation of the inductive definition of $T$ for clarification: 


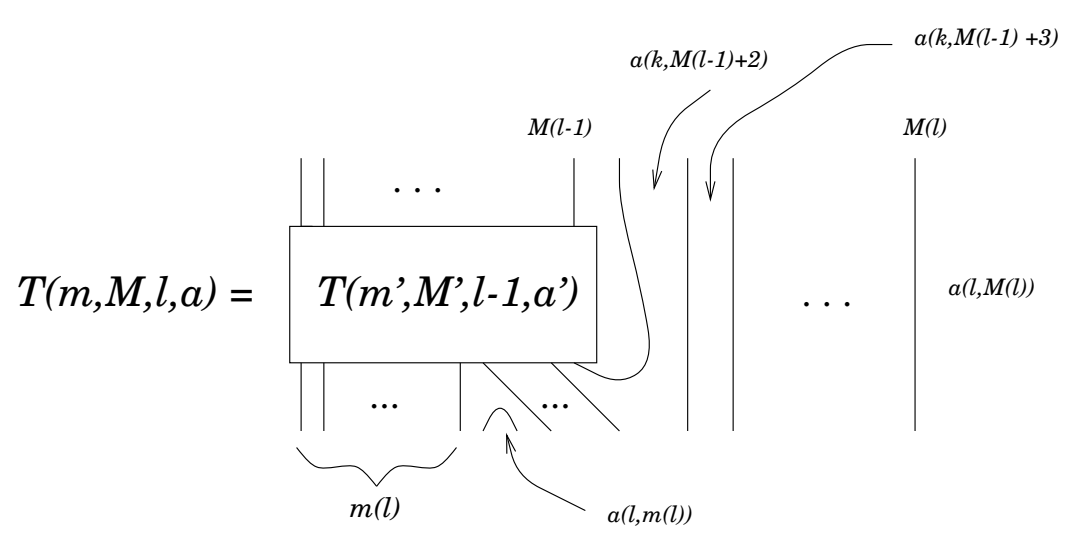

These maps are clearly well defined and inverses and thus we have:

Theorem 1. The maps $\psi$ and $T$ establish a one-to-one correspondence between $n+1$-blocks and Kauffman $(k, n)$-diagrams. Moreover when this correspondence is restricted to reduced $(k, n+1)$-blocks, it yields a one-to-one correspondence between reduced $(k, n+1)$-blocks and Kauffman $(k, n)$-diagrams with the added condition that the sum of labels of any two adjacent pieces is $\geq k$.

Proof. This follows from the inductive definitions of $T$ and $\psi$.

Next we need a correspondence well known to combinatorialists. We define a planar $(k, n)$-tree to be a rooted planar tree with $n$ edges and vertices labeled by integers in the interval $[0, \mathrm{k}]$.

Theorem 2. There is a one-to-one correspondence between Kauffman $(k, n)$-diagrams and planar $(k, n)$-trees. Furthermore, in this correspondence, labels of adjacent pieces of the Kauffman diagram label neighboring vertices on the corresponding planar tree.

Proof. We use the well-known bijection between Kauffman (0, $n)$-diagrams and planar $(0, n)$-trees, [St, Exercise 6.19 e \& o, p. 256]. In this bijection, the vertices of a planar tree are in one-to-one correspondence with the pieces of the corresponding Kauffman diagram. Thus the bijection extends to a bijection between Kauffman $(k, n)$-diagrams and planar $(k, n)$-trees. The result follows from the fact that labels of adjacent vertices of a tree get sent to adjacent pieces of the Kauffman diagram and vice-versa. For clarity, we include the following picture that illustrates the correspondence between a Kauffman (2,5)-diagram (dotted lines) and a planar (2,5)-tree (solid lines, the tree is turned on its side with the root at the right): 


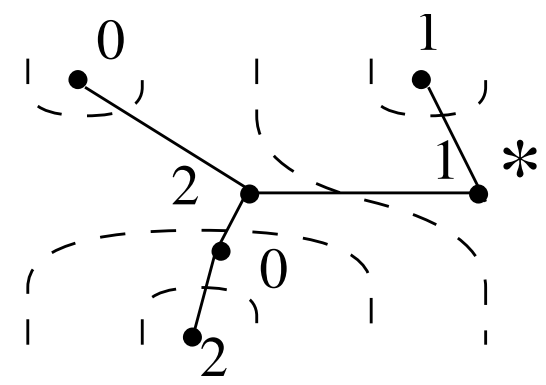

Theorem 3. There is a one-to-one correspondence between reduced $(k, n+$ $1)$-blocks and $(k, n)$ planar trees for which the sum of labels of any two adjacent vertices is $\geq k$.

Proof. This follows from Theorems 1 and 2, and the fact that reduced $(k, n)$ blocks are $(k, n)$ blocks with the added condition that

b3. $a(i, j)+a(i, j-1) \geq k$

$$
a(i, j)+a(i+1, j+1) \geq k \text {. }
$$

Corollary 3.1. There is a one-to-one correspondence between reduced $(k, n+1)$-blocks and $(k, n)$ planar trees for which the sum of labels of any two adjacent vertices is $\leq k$.

Proof. Replacing every lable of a vertex by $k$ minus that label gives a oneto-one correspondence between

$\{(k, n)$ planar trees with the sum of labels of any two adjacent vertices

$$
\begin{gathered}
\geq k\} \\
\text { and }
\end{gathered}
$$

$\{(k, n)$ planar trees with the sum of labels of any two adjacent vertices $\leq k\}$.

The result then follows from Theorem 3 .

We are now ready to complete the plan of counting the number of reduced words described at the end of Section 3.

\section{Counting.}

We have established a one-to-one correspondence between the reduced $(k, n)$ blocks and $(k, n)$-planar trees for which the sum of labels of any two adjacent vertices is $\leq k$. Using this correspondence we will now show that the generating function

$$
T(x)=1+\sum_{i=1}^{\infty}\left|S_{k, i}\right| x^{i}
$$


satisfies the relation:

$$
x T^{k+2}=T-1 .
$$

Let $A_{i}^{n}$ be the number of planar $(k, n-1)$ trees with

1) the root vertex labeled by $i$,

2) the sum of labels of any two adjacent vertices $\leq k$.

Thus $n$ will index the number of vertices on the tree. Let $A_{i}(x)=\sum_{n=1}^{\infty} A_{i}^{n} x^{n}$, and let $A(x)=\sum_{i=0}^{k} A_{i}(x)$. Writing $A(x)=\sum_{i=1}^{\infty} a_{i} x^{i}$ we have from Corollary 3.1 that $a_{i}$ is the number of reduced $(k, i)$-blocks.

Recall (Definition 5) that a word $w \in S_{k, n}$ has the form:

1) $w=1$,

or

2) $w=\prod_{i=1}^{d} w_{i}$ with a) the $w_{i}$ reduced blocks,

b) $\min \left(w_{i+1}\right)>\max \left(w_{i}\right)$.

The elements $w=\prod_{i=1}^{d} w_{i} \in S_{k, n}$ fall into two disjoint categories:

i) $\max \left(w_{d}\right)<n-1$, in which case $w$ can be any element of $S_{k, n-1}$,

ii) $\max \left(w_{d}\right)=n-1, \min \left(w_{d}\right)=j$, in which case $w$ can be described as the product of an element of $S_{k, j}$ (i.e., $\prod_{i=1}^{d-1} w_{i}$ ) and a reduced block $w_{d}$ with $\max \left(w_{d}\right)=n-1$ and $\min \left(w_{d}\right)=j$.

Thus we have the relation for $n>1$,

$$
\left|S_{k, n}\right|=\left|S_{k, n-1}\right|+\sum_{j=1}^{n-1}\left|S_{k, j}\right| a_{n-j} .
$$

The above and the fact that $\left|S_{1, k}\right|=1$, translate to the generating function equation:

$$
\sum_{i=1}^{\infty}\left|S_{k, i}\right| x^{i}=x \sum_{i=1}^{\infty}\left|S_{k, i}\right| x^{i}+\left(\sum_{i=1}^{\infty}\left|S_{k, i}\right| x^{i}\right) A(x)+x .
$$

Thus

$$
T(x)-1=x(T(x)-1)+(T(x)-1) A(x)+x
$$

and solving for $T(x)$ and $A(x)$ respectively yields:

$$
\begin{aligned}
& T(x)=\frac{1-A(x)}{1-A(x)-x}, \\
& A(x)=1-\frac{x T(x)}{T(x)-1} .
\end{aligned}
$$

Consider a tree counted by $A_{i}^{n}$. The root is labeled by $i$ and the first vertex (as the tree is traced clockwise from the root), if one exists, is labeled by $l$ : 


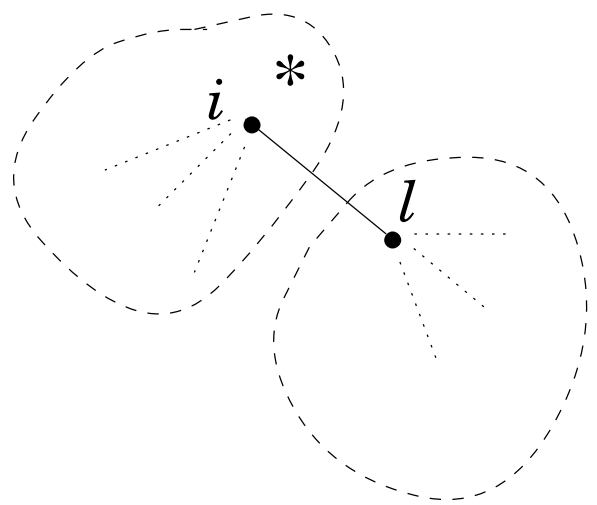

The tree can be thought of as the joining of two trees (the dotted circles), one with root labeled $i$ and the other with root labeled $l$ for some $l$ with $i+l \leq k$. We therefore have the recursion:

$$
A_{i}^{n}=\sum_{j=1}^{n-1} A_{i}^{j}\left(\sum_{l=0}^{k-i} A_{l}^{n-j}\right) \quad \text { for } n>0 .
$$

In terms of $A_{i}(x)$, this relation becomes

$$
A_{i}(x)=x+\sum_{l=0}^{k-i} A_{i}(x) A_{l}(x)
$$

with the initial $x$ to allow for the tree with exactly one vertex. Setting $L_{i}(x)=1-\sum_{l=0}^{i} A_{l}(x),(3)$ becomes

$$
\begin{gathered}
\left(1-L_{0}(x)\right) L_{k}(x)=x \\
\left(L_{i-1}(x)-L_{i}(x)\right) L_{k-i}(x)=x \quad 1 \leq i \leq k .
\end{gathered}
$$

For convenience of notation, we will omit the dependence on $x$ whenever possible (i.e., we will denote $L(x)$ by $L$ ). Rewriting (5) we have

$$
L_{i} L_{k-i}=L_{i-1} L_{k-i}-x .
$$

Evaluating (6) at the two values $i=j$ and $i=k-(j-1)$ gives

$$
L_{j} L_{k-j}=L_{j-1} L_{k-j}-x=\left(L_{k-(j-1)} L_{j-1}+x\right)-x=L_{k-(j-1)} L_{j-1},
$$

and thus $L_{j} L_{k-j}=L_{0} L_{k}$ for $1 \leq j \leq k$. Using (4) to express $L_{0} L_{k}$ in terms of $L_{k}$ we have

$$
L_{i} L_{k-i}=L_{k}-x
$$

It follows from (6) that

$$
L_{i-1} L_{k-i}=L_{k}-x+x=L_{k} .
$$


Combining (7) and (8) yields

$$
L_{i}=\left(\frac{L_{k}-x}{L_{k}}\right) L_{i-1}
$$

and thus

$$
L_{i}=\left(\frac{L_{k}-x}{L_{k}}\right)^{i} L_{0}=\left(\frac{L_{k}-x}{L_{k}}\right)^{i+1} .
$$

For $i=k$ the above becomes

$$
L_{k}=\left(\frac{L_{k}-x}{L_{k}}\right)^{k+1} .
$$

Combining (10),(2) and $L_{k}=1-A$ implies that $T$, the generating function for $\left|S_{k, n}\right|$, satisfies the relation:

$$
x T^{k+2}=T-1 .
$$

\section{6. $F C_{k, n}$.}

We now define the Fuss-Catalan algebras of [BiJo1]. Recall that in a Kauffman $n$-diagram we had labeled the boundary points $1,2, \ldots, 2 n$ as we traveled clockwise around the picture starting at the top left, ending at the bottom left.

Definition 8 ([BiJo1]). Label the boundary points of the Kauffman $(k+$ 1) $n$-diagrams clockwise by the labels:

$$
a_{0}, a_{1}, a_{2} \ldots a_{k}, a_{k}, a_{k-1} \ldots a_{1}, a_{0}, a_{0} \ldots
$$

(Thus boundary point $2(k+1) n$ is labeled by $a_{0}$.) Define $\mathcal{B}\left(k, n, a_{0}, a_{1}, \ldots a_{k}\right)$ to be the set of all Kauffman $(k+1) n$-diagrams such that for each $i$, the boundary points $i$ and $J(i)$ have the same "a" label. (This is equivalent to $i+J(i) \equiv 1 \bmod 2(k+1)$.)

Definition 9 ([BiJo1]). Let $F C_{k, n}\left(\alpha_{0}, \alpha_{1} \ldots \alpha_{k}\right), \alpha_{i} \in \mathbb{C}-0$ be the complex linear span of $\mathcal{B}\left(k, n, a_{0}, a_{1} \ldots a_{k}\right)$, with the following multiplicative structure:

Given $D_{1}, D_{2} \in \mathcal{B}(k, n)$. Define $D_{3} \in \mathcal{B}(k, n)$ as follows: stack $D_{2}$ on top of $D_{1}$ so that the boundary points at the bottom of $D_{2}$ are aligned with those at the top of $D_{1}$. Fuse the curves of $D_{2}$ and $D_{1}$ together at these boundary points. Remove all closed loops and let $D_{3}$ be the resulting diagram.

We define

$$
D_{1} D_{2}=\left(\alpha_{0}\right)^{n_{0}}\left(\alpha_{1}\right)^{n_{1}} \ldots\left(\alpha_{k}\right)^{n_{k}} D_{3}
$$

where $n_{i}$ is the number of removed closed loops of curves of type " $a_{i}$ ". 
Note. The notation differs slightly from [BiJo1] in that the labels of the curves are $a_{i}$ whereas the scalars associated to loops of type " $a_{i}$ " are the $\alpha_{i}$. In [BiJo1] $a_{i}$ represented both these quantities. We do this to clarify that the types " $a_{i}$ " are all distinct whereas the scalars $\alpha_{i}$ need not be. From now on we will refer to $\mathcal{B}\left(k, n, a_{0}, a_{1} \ldots a_{k}\right)$ as $\mathcal{B}(k, n)$, suppressing the labeling set of the vertices.

Definition 10 ([BiJo1]). We define a *-operation on $F C_{k, n}\left(\alpha_{0}, \alpha_{1}, \ldots \alpha_{k}\right)$, $\alpha_{i} \in \mathbf{R}$ as follows: if $D \in F C_{k, n}$ is a diagram, denote by $D^{*}$ the diagram obtained by the reflection of $D$ over the top boundary. Thus the top (resp. bottom) boundary of $D^{*}$ is the bottom (resp. top) boundary of $D$. Extend the ${ }^{*}$-operation to all of $F C_{k, n}$ by conjugate linearity.

We note that $D^{* *}=D$ and $\left(D_{1} D_{2}\right)^{*}=D_{2}^{*} D_{1}^{*}$ (since the parameters are real) and so $*$ is a conjugate linear involution of $F C_{k, n}$.

Definition 11. Let ${ }_{a} e_{i}, 1 \leq i \leq n-1,0 \leq a \leq k$ denote the idempotent in $F C_{k, n}$ that is $\frac{1}{a c_{i}}$ times the following picture:

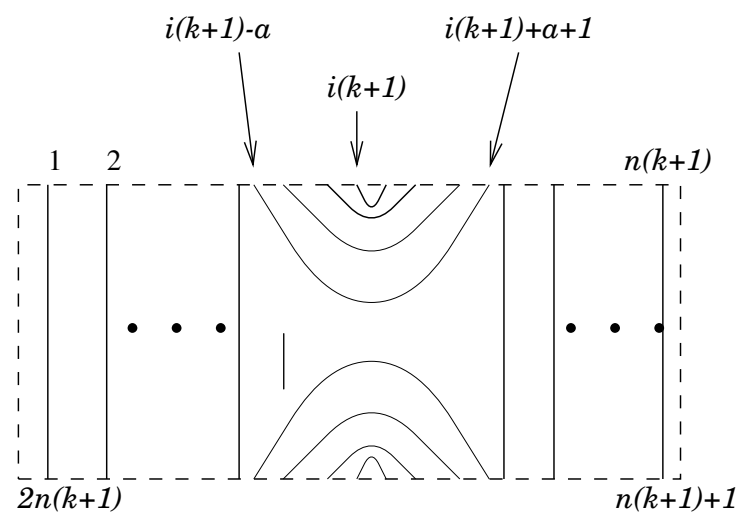

where

$$
{ }_{a} c_{i}= \begin{cases}\alpha_{0} \alpha_{1} \ldots \alpha_{a} & \text { for i even } \\ \alpha_{k-a} \alpha_{k-a+1} \ldots \alpha_{k} & \text { for i odd }\end{cases}
$$

We want to show that $F C_{k, n}$ is generated by the set $\left\{1,{ }_{a} e_{i}\right\}$. To do so, we use the following notation and lemma:

Denote by a box labeled $i$ a Kauffman diagram consisting of $i$ vertical strings:

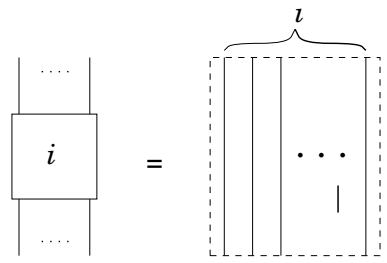


Lemma 5. Given $K \in \mathcal{B}(k, n)$ with corresponding planar function J. Suppose that $|J(i)-i|<2(k+1)$ for all $i \in S=\left\{i_{0}, i_{0}+1, \ldots i_{0}+k\right\}$. Then up to horizontal and/or vertical symmetry (i.e., reflection in the $x$ and/or $y$ axes) there exists an integer $c, 0 \leq c \leq k+1$ such that $K$ can be drawn in one of the following two ways:

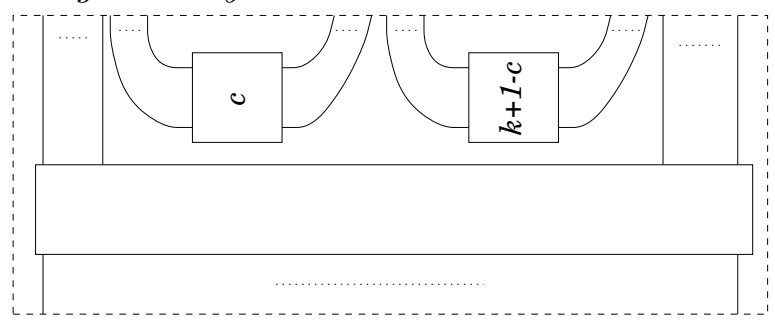

or

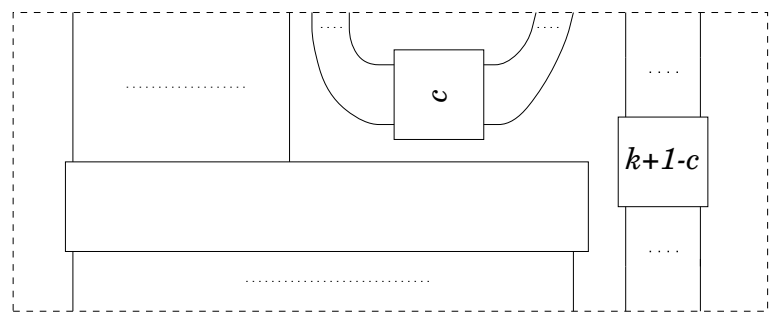

Proof. Choose $i$ so that $i \in S$ and $|i-J(i)|$ is maximal ( $i$ is not necessarily unique). Let us assume $J(i)<i$, an identical argument holds otherwise. Because there are only two boundary points of each type between $J(i)$ and $i$, we must have $K$ of the form:
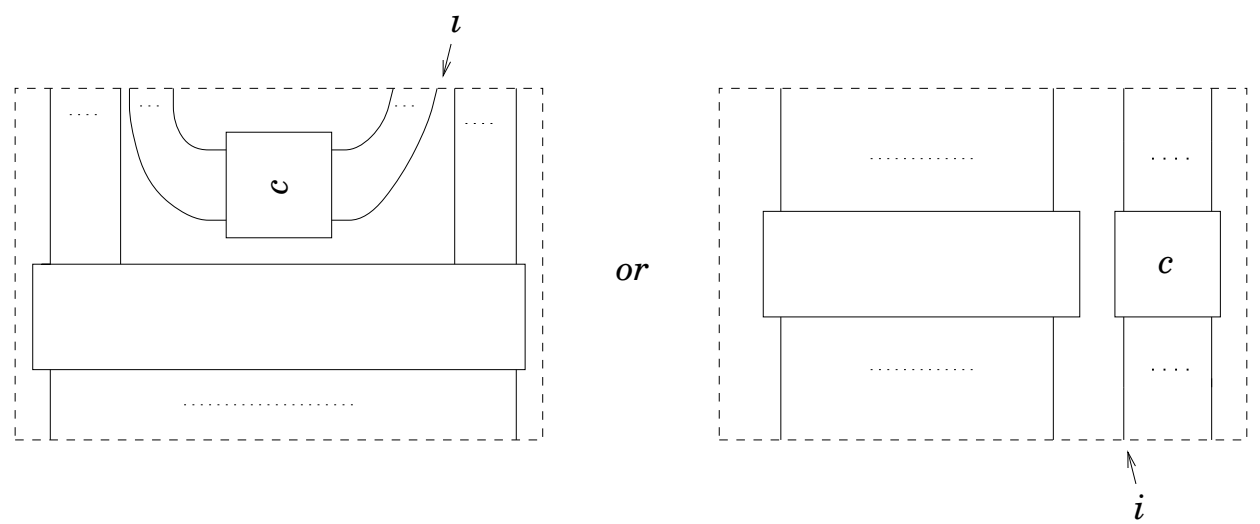

with $c=(i-J(i)+1) / 2$. If $|i-J(i)|=2(k+1)-1$ we are done (since $c=k+1)$. Otherwise either $J(i)-1$ or $i+1$ is in $S$. By symmetry let us assume $i+1 \in S$. We then have $J(i+1)>(i+1)$ (otherwise $J(i+1)<J(i)$ contradicting the choice of $i)$, and $J(i+1)-(i+1)<2(k+1)$. Again, since 
there are only two boundary points of each type between $i+1$ and $J(i+1)$ we are left with one of the two pictures we wanted.

Armed with the above lemma we have the following:

Theorem 4. The set $\left\{1,{ }_{a} e_{i}\right\}$, with $1 \leq i \leq n-1$ and $0 \leq a \leq k$ is a generating set for $F C_{k, n}$.

Proof. We induct on $n$. The result is clear for $n=1,2$. We first show that all elements of $\mathcal{B}(k, n) \subset F C_{k, n}$ satisfy the conclusion of Lemma 5. Consider a pair of points $(i, J(i)), i<J(i)$ with $|i-J(i)|$ minimal among all pairs $(j, J(j))$ with $|j-J(j)| \geq 2(k+1)$. If no such $i$ exists then the hypothesis for Lemma 5 is satisfied for any string of $k+1$ consecutive boundary points. If an $i$ does exist then all the points $i \leq j \leq J(i)$, will have $i \leq J(j) \leq J(i)$ (by planarity of $J)$. This contradicts the choice of $i$ unless $|j-J(j)|<2(k+1)$ in which case we again have at least $k+1$ points in a row with $|j-J(j)| \leq 2 k$ and thus we can apply Lemma 5 .

Up to reflection in the $x$ and/or $y$ axis, we then have one of two possibilities for the diagram of an arbitrary element of $\mathcal{B}(k, n)$. The first:

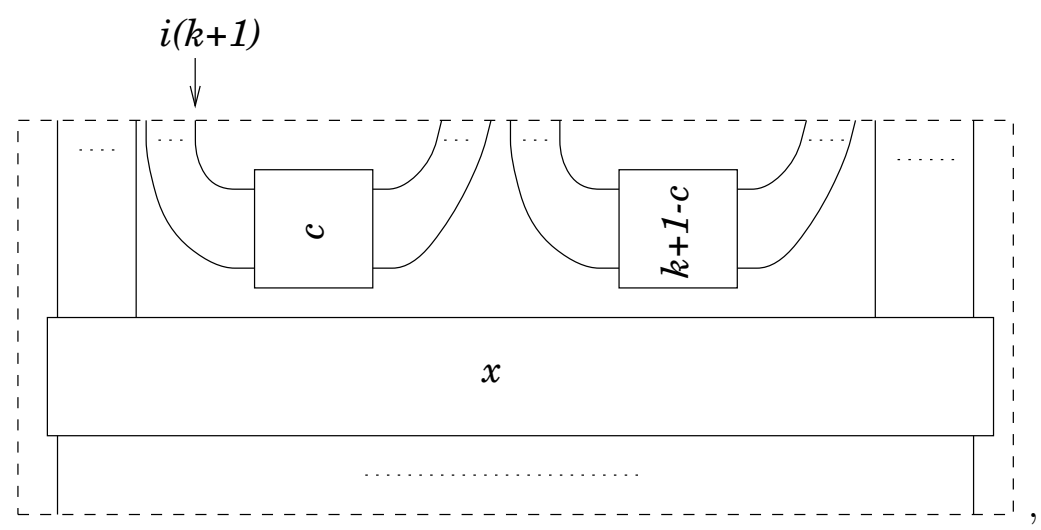

can be rewritten as 


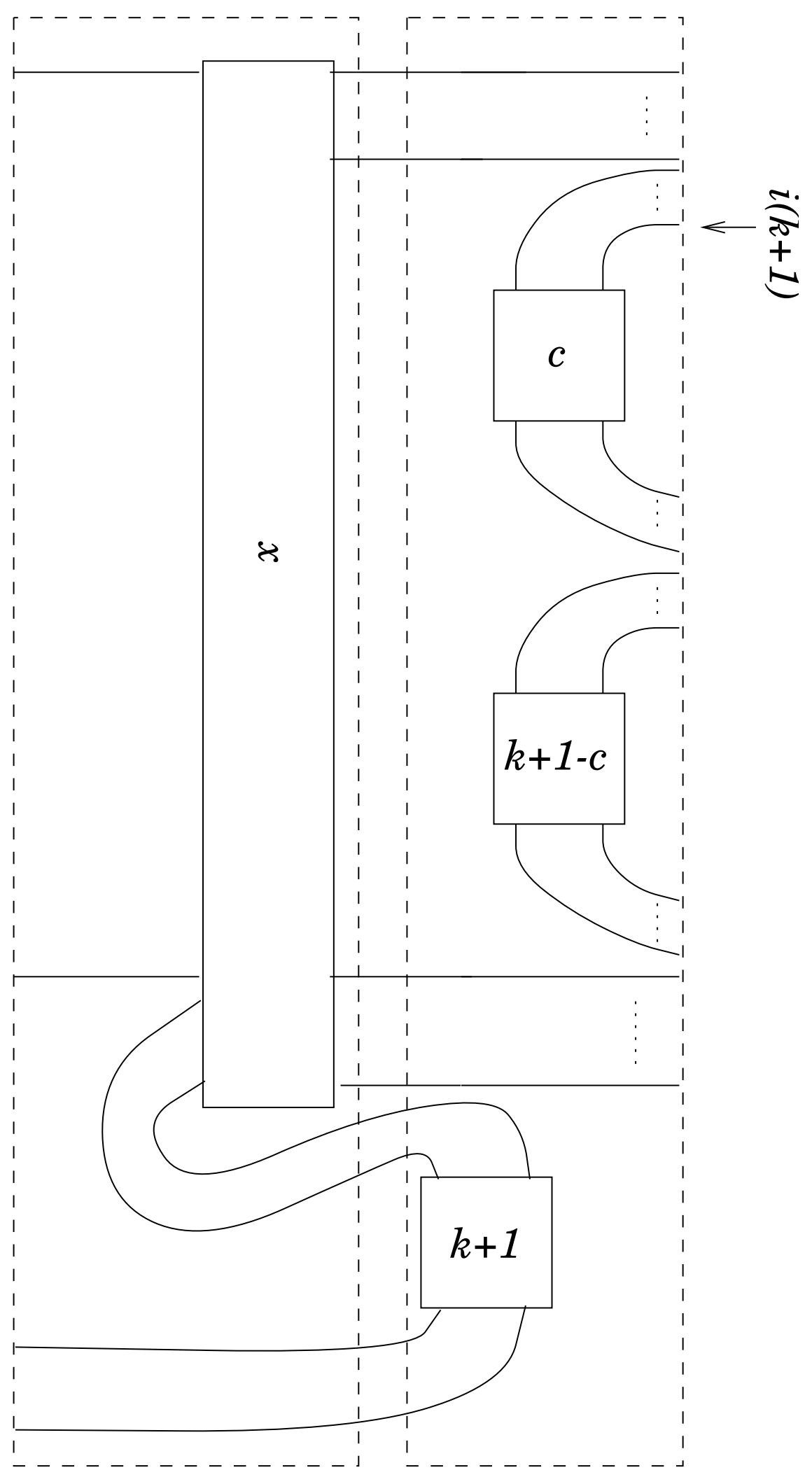


The upper dotted diagram is just a scalar multiple of

$$
\left({ }_{k} e_{n-1} \cdot{ }_{k} e_{n-2} \cdots{ }_{k} e_{i+1}\right)_{c-1} e_{i}
$$

while the lower dotted diagram is an element of $F C_{k, n-1}$ sitting inside $F C_{k, n}$ and thus by the induction hypothesis the whole diagram is a product of elements in $\left\{1,{ }_{i} e_{a}\right\}$. Similarly the second possible picture for an element in $\mathcal{B}(k, n)$ : 


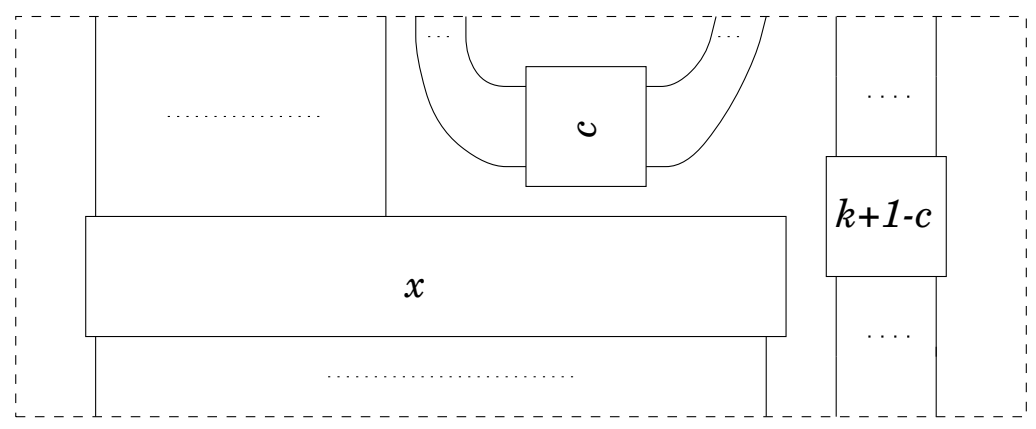

can be written as

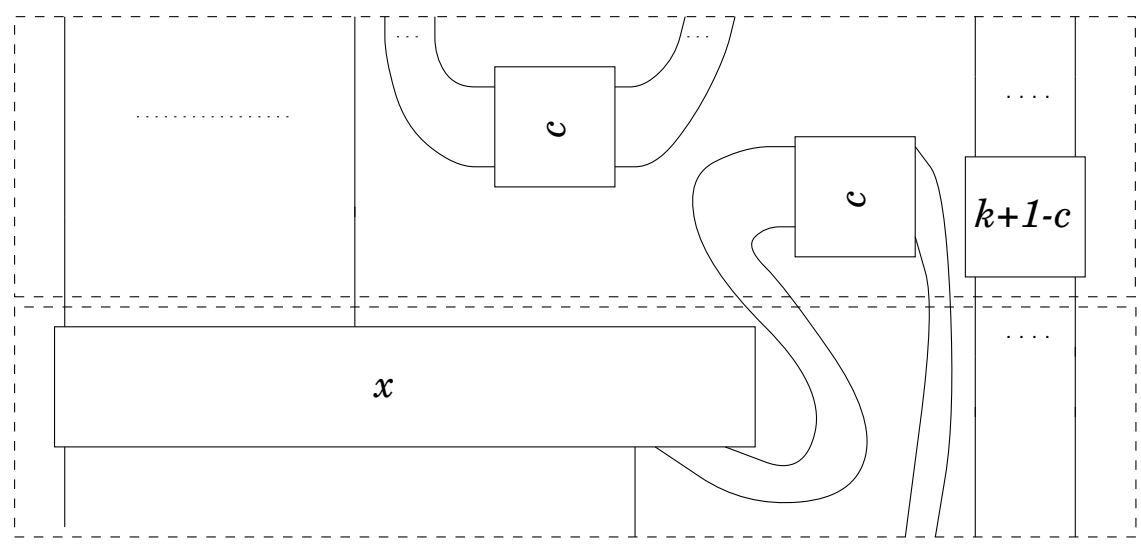

The upper dotted diagram is just a scalar multiple of

$$
c-1 e_{n-1}
$$

while once again the lower dotted diagram is an element of $F C_{k, n-1}$ sitting inside $F C_{k, n}$. Once again the induction hypothesis allows us to conclude that the diagram is generated by a product of elements in $\left\{1,{ }_{a} e_{i}\right\}$. Similar arguments hold for $x$ and/or $y$ axis reflection of the diagrams.

Theorem 5 ([BiJo1 $]) \cdot \operatorname{dim} F C_{k, n}=\frac{1}{(k+1) n+1}\left(\begin{array}{c}(k+2) n \\ n\end{array}\right)=c_{n}^{k+2}$ and $G(x)=$ $\sum_{n=0}^{\infty} c_{n}^{k+2} x^{n}$ satisfies:

$$
G(x)=x(G(x))^{k+2}+1 .
$$

For a nice combinatorial proof of the above see $[\mathbf{H e}]$.

\section{7. $A A_{k, n}$ isomorphic to $F C_{k, n}$.}

Theorem 6. The algebras $A A_{k, n}\left(\alpha_{0}, \alpha_{1}, \ldots \alpha_{k}\right)$ and $F C_{k, n}\left(\alpha_{0}, \alpha_{1}, \ldots \alpha_{k}\right)$ are isomorphic. In addition, $S_{k, n}$ is a basis for $A A_{k, n}\left(\alpha_{0}, \alpha_{1}, \ldots \alpha_{k}\right)$. 
Proof. The map $\phi: A A_{k, n}\left(\alpha_{0}, \alpha_{1}, \ldots \alpha_{k}\right) \rightarrow F C_{k, n}\left(\alpha_{0}, \alpha_{1}, \ldots \alpha_{k}\right)$ given by

$$
\phi\left({ }_{a} E_{i}\right)={ }_{a} e_{i}
$$

is a homomorphism onto $F C_{k, n}$ due to the universality of the abstract algebra and the fact that the generators of $F C_{k, n}$ (Theorem 4) satisfy the same relations as the abstract ones. Since the generating functions for the dimension of $F C_{k, n}\left(\alpha_{0}, \alpha_{1}, \ldots \alpha_{k}\right)$ (Theorem 5) and for $\left|S_{k, n}\right|$ (Section 5) are the same, we have that the dimension of $F C_{k, n}$ is $\left|S_{k, n}\right|$. We deduce that the dimension of $A A_{k, n}$ is equal to $\left|S_{k, n}\right|$ since it is both $\geq\left|S_{k, n}\right|$ (since $\phi$ is onto) and $\leq\left|S_{k, n}\right|$ (Section 3). It follows that $\phi$ is an isomorphism and that $S_{k, n}$ is a basis for $A_{k, n}\left(\alpha_{0}, \alpha_{1}, \ldots \alpha_{k}\right)$.

\section{The Structure of $F C_{k, n}$.}

Lemma 6 ([BiJo1]). The algebras $F C_{k, n}\left(\alpha_{0}, \alpha_{1} \ldots \alpha_{k}\right)$ are semisimple for an open dense (even Zariski open) subset of parameters $\left(\alpha_{0}, \alpha_{1} \ldots \alpha_{k}\right) \in$ $\mathbb{C}^{k+1}$.

We now describe the structure of $F C_{k, n}$ in the case when $F C_{k, n}$ is semisimple (the generic case) giving the Bratteli diagram for the inclusion $F C_{k, 0}$ $\subset F C_{k, 1} \subset F C_{k, 2} \subset \ldots$ In essence, this work was done in [BiJo1, Section 3], though only explicitly described for $k=1$ (i.e., for 2 parameters $\alpha_{0}, \alpha_{1}$ ). We describe this structure, stating, but not proving, those results that are straightforward generalizations of arguments given in [BiJo1].

Definition 12 ([BiJo1]). A through string on a diagram $D \in \mathcal{B}(k, n)$ is a curve that connects a point on the top of $D$ to one on the bottom. Let $t(D)$ be the number of through strings of $D$.

Definition 13 ([BiJo1]). The middle pattern on a diagram $D \in \mathcal{B}(k, n)$ is the well defined word, denoted by $m(D)$, obtained by reading from left to right the labels " $a_{i}$ " of the through strings of $D$.

We give an example to clarify:

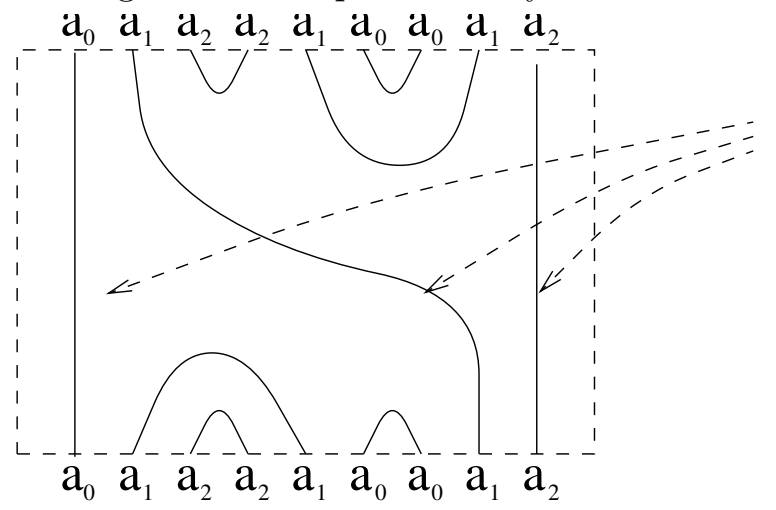

through strings

middle pattern: $a_{0} a_{1} a_{2}$ 
So far, we have dealt with planar diagrams that have the same number of boundary points on the top as on the bottom. We now define a more general picture:

Definition 14 ([BiJo1]). Let $D_{p l}(p, q)$ be the set of diagrams with $p$ boundary points on the bottom and $q$ on the top with non-intersecting curves (strings) connecting distinct boundary points.

(The $p l$ in $D_{p l}$ refers to the "planarity" or non-intersecting property of the diagram.)

We note that an element of $D_{1} \in D_{p l}(p, q)$ can be multiplied by an element in $D_{2} \in D_{p l}(q, r)$ as long as the labeling of the top of $D_{1}$ coincides with the labeling of the bottom of $D_{2}$. Thus an element $D \in \mathcal{B}(k, n)$ can be written uniquely as the composition of $D_{\text {in }} \in D_{p l}((k+1) n, t(D))$, the initial part, and $D_{\text {fin }} \in D_{p l}(t(D),(k+1) n)$, the final part, where the top of $D_{i n}$ and the bottom of $D_{\text {fin }}$ are labeled by $m(D)$. (Of course the top of $D_{\text {fin }}$ and the bottom of $D_{i n}$ are labeled by $\left.a_{0}, a_{1}, a_{2} \ldots a_{k}, a_{k}, a_{k-1} \ldots a_{1}, a_{0}, a_{0} \ldots\right)$.

We include an example to clarify:

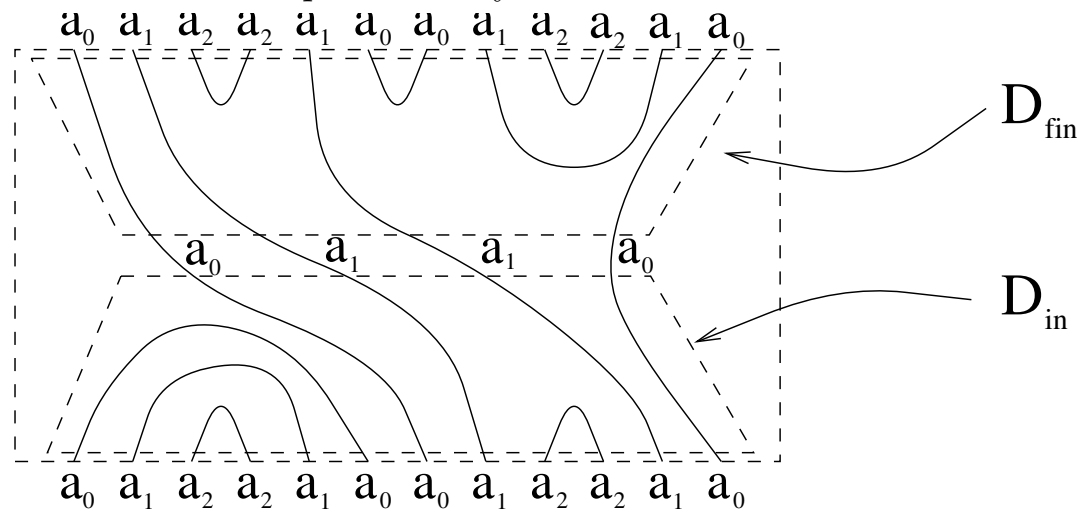

For two words $w, w^{\prime}$ we define $w \leq w^{\prime}$ if $w$ is obtained from $w^{\prime}$ by removing some (or no) letters of $w^{\prime}$. Define $v_{n}$ to be the following word with $(k+1) n$ letters:

$$
a_{0} a_{1} a_{2} \ldots a_{k} a_{k} a_{k-1} \ldots a_{1} a_{0} a_{0} \ldots \ldots a_{0}\left(\text { or } a_{k}\right) \text {. }
$$

The following is a straightforward observation:

Lemma 7. The word $w$ appears as a middle pattern for elements in $F C_{k, n}$ if and only if $w \leq v_{n}$ and $w \equiv v_{n}$ when reduced using the relations $a_{i}^{2}=$ $1,0 \leq i \leq k$.

Note that the smallest such middle pattern is $\phi$ for $n$ even and $a_{0} a_{1} \ldots a_{k}$ for $n$ odd.

Definition 15 ([BiJo1]). Let $I_{t}$ be the two sided ideal of $F C_{k, n}$ spanned by diagrams with at most $t$ through strings. 
For obvious parity reasons, for $n$ even we have $I_{2 t}=I_{2 t+1}$ and

$$
\{0\} \subset I_{0} \subset I_{2} \subset I_{4} \subset \cdots \subset I_{n(k+1)}=F C_{k, n}\left(\alpha_{0}, \alpha_{1} \ldots \alpha_{k}\right) .
$$

For $n$ odd we have

$$
\{0\}=I_{0}=I_{1}=I_{2}=\cdots=I_{k}, \quad I_{k+2 l+1}=I_{k+2 l+2}
$$

and

$$
\{0\} \subset I_{k+1} \subset I_{k+3} \subset I_{k+5} \subset \cdots \subset I_{n(k+1)}=F C_{k, n}\left(\alpha_{0}, \ldots \alpha_{k}\right) .
$$

Thus, in the semisimple case, to describe the structure of $F C_{k, n}$ it will suffice to describe the appropriate algebras $I_{t+2} / I_{t}$, where $t$ is the same parity as $(k+1) n$.

Given $D \in \mathcal{B}(k, n)$ define the projection $p_{M}=\frac{1}{c_{0}} M M^{*}$ where $M=D_{i n}$ and $c_{0} M M^{*}=M M^{*} M M^{*}$.

Lemma 8 ([BiJo1]). The projection $p_{M}$ has the following properties:

1) $p_{M} F C_{k, n} p_{M} \equiv \mathbb{C} p_{M} \bmod I_{t(D)-2}$.

2) Given $M_{1}, M_{2}$ initial parts of two diagrams with distinct middle patterns of length $t$, then $p_{M_{1}} E p_{M_{2}} \equiv 0 \bmod I_{t-2}$ for $E$ a diagram in $F C_{k, n}$.

3) Given $M_{1}$ and $M_{2}$ initial parts of two diagrams with the same middle pattern, we have $M_{1}=c D M_{2}$ for some $D \in F C_{k, n}, c \in \mathbb{C}$.

Definition 16 ([BiJo1]). Given a word $w$ in the alphabet $\left\{a_{0}, a_{1}, \ldots a_{k}\right\}$, define $\left[\begin{array}{l}n \\ w\end{array}\right]$ to be the number of distinct initial parts of the diagrams in $\mathcal{B}(k, n)$ having middle pattern $w$. (Define $\left[\begin{array}{l}n \\ w\end{array}\right]=0$ if $w$ is not a middle pattern.)

The above work leads us to the following:

Lemma 9 ([BiJo1]). If $w$ is a middle pattern of length $t$ and $M$ is an initial part of a diagram with middle pattern $w$, then the dimension of the left ideal $p_{M}\left(I_{t} / I_{t-2}\right)$ is $\left[\begin{array}{c}n \\ w\end{array}\right]$.

\section{Lemma 10 ([BiJo1] $)$.}

$$
I_{t} / I_{t-2}=\bigoplus_{w}\left(I_{t} / I_{t-2}\right) p_{M}\left(I_{t} / I_{t-2}\right),
$$

where the sum is over all middle patterns of length $t$, and $M$ is an initial part of a diagram with middle pattern $w$ (one $M$ for each $w$ ).

Theorem 7 ([BiJo1] $)$. If $\left(\alpha_{0}, \alpha_{1} \ldots \alpha_{k}\right)$ are such that $F C_{k, n}\left(\alpha_{0}, \alpha_{1} \ldots \alpha_{k}\right)$ is semisimple, we have

$$
F C_{k, n}\left(\alpha_{0}, \alpha_{1} \ldots \alpha_{k}\right) \cong \bigoplus_{w:\left[\begin{array}{c}
n \\
w
\end{array}\right]>0} M_{\left[\begin{array}{l}
n \\
w
\end{array}\right]}(\mathbb{C}),
$$

where the sum is over all middle patterns $w$ of diagrams in $F C_{k, n}$, and the summands are matrix algebras over $\mathbb{C}$ of dimension $\left[\begin{array}{l}n \\ w\end{array}\right]$. 
With this decomposition we can describe the irreducible representations. We need the following definition:

Definition 17 ([BiJo1]). Given $w$ a word $t$ letters long in the alphabet $\left\{a_{0}, a_{1} \ldots a_{k}\right\}$, with $\left[\begin{array}{l}n \\ w\end{array}\right]>0$. Let $V_{w}$ be the complex vector space of dimension $\left[\begin{array}{l}n \\ w\end{array}\right]$ having as basis distinct initial parts $M$ of diagrams in $\mathcal{B}(k, n)$ with middle pattern $w$. For $D \in \mathcal{B}(k, n)$ define the representation $\pi_{w}$ of $F C_{k, n}$ (extended by linearity) on $V_{w}$ as follows:

$$
\pi_{w}(D) M= \begin{cases}D M, & \text { if } D M \text { has } t \text { through strings, } \\ 0, & \text { otherwise, }\end{cases}
$$

for all $M \in V_{w}$ (where as usual we count a closed loop of type " $a_{i}$ " as a scaling factor $\left.\alpha_{i}\right)$.

With the above notation, we have:

Theorem 8 ([BiJo1]). If $\left(\alpha_{0}, \alpha_{1} \ldots \alpha_{k}\right)$ are such that $F C_{k, n}\left(\alpha_{0}, \alpha_{1} \ldots \alpha_{k}\right)$ is semisimple, then all the representations $\pi_{w}$ are irreducible and any irreducible representation of $F C_{k, n}$ is equivalent to a $\pi_{w}$.

A description of the Bratteli diagram turns out to be quite simple for the following more general situation. Given an infinite word $w=\prod_{n=0}^{\infty} t_{n}, t_{i} \in$ $\left\{a_{0}, a_{1} \ldots a_{k}\right\}$, let $w_{i}=\prod_{n=0}^{i} t_{n}$. Define $A_{w_{i}}\left(\alpha_{0}, \alpha_{1} \ldots \alpha_{k}\right)$ to be the complex algebra with basis the set of Kauffman $n$ diagrams whose top and bottom row are labeled (both from left to right) by $w_{i}$, and whose curves connect boundary points of the same label (again, loops labeled by " $a_{i}$ " are replaced by a scalar factor " $\alpha_{i}$ "). Define $\pi_{v}^{(i)}$ to be the irreducible representation of $A_{w_{i}}$ whose vector space consists of all initial parts of diagrams with middle pattern $v$. Then we have:

Theorem 9 ([BiJo1]). Suppose the algebras $A_{w_{i}}$ defined above are semisimple. Let $v$ be a word in the alphabet $\left\{a_{0}, a_{1}, \ldots a_{k}\right\}$, let $t \in\left\{a_{0}, a_{1}, \ldots a_{k}\right\}$ and suppose that $v t$ is a middle pattern for $A_{w_{i+1}}$. Then

$$
\left.\pi_{v t}^{(i+1)}\right|_{A_{w_{i}}} \equiv \begin{cases}\pi_{v}^{(i)} \bigoplus \pi_{v t t}^{(i)} & \text { if } t_{i+1}=t \\ \pi_{v t t_{i+1}}^{(i)} & \text { otherwise. }\end{cases}
$$

This theorem determines the Bratteli diagram for $A_{w_{0}} \subset A_{w_{1}} \subset A_{w_{2}} \subset$ .... The following sentence is the rule that describes the above theorem [BiJo1, p. 113]: "The words on the ith row are certain subwords of $w_{i}$. To connect the $\mathrm{i}$-th row to the $(\mathrm{i}+1)$ th, add $t_{i+1}$ to all words and take it away, if possible".

If we now return to the special case of $F C_{k, n}$, where our infinite word is

$$
w=a_{0} a_{1} a_{2} \ldots a_{k} a_{k} a_{k-1} \ldots a_{1} a_{0} a_{0} \ldots,
$$

we have $F C_{k, n}=A_{w_{(k+1) n}}$, that is, we are looking for the inclusion every $k+1$ steps. Recall that $F C_{k, n}$ is isomorphic to a direct sum of matrix 
algebras indexed by the distinct middle patterns that occur for diagrams in $F C_{k, n}$. Thus the vertices of the Bratteli diagrams at the $n$-th level are indexed by these middle patterns as well. Let $\pi_{w}^{n}$ denote the representation of $\pi_{w}$ described in Definition 17 applied to the algebra $F C_{k, n}$. Let us denote by $N_{\pi_{w}^{n}, \pi_{w^{\prime}}^{n+1}}$, the number of edges in the Brattelli diagram for $F C_{k, n} \subset F C_{k, n+1}$ between the vertex indexed by $w$ in $F C_{k, n}$ and that of $w^{\prime}$ in $F C_{k, n+1}$. Alternatively, $N_{\pi_{w}^{n}, \pi_{w^{\prime}}^{n+1}}$ is the number of times $\pi_{w}^{n}$ occurs in $\left.\pi_{w^{\prime}}^{n+1}\right|_{F C_{k, n}}$. Using Theorem 9, we can describe the Bratteli diagram for $F C_{k, n} \subset F C_{k, n+1} \subset F C_{k, n+2}$ :

Lemma 11 ([BiJo1]). Assume that $F C_{k, n}$ are semisimple. Let $w$ (resp. $\left.w^{\prime}\right)$ be a middle pattern for $F C_{k, n}$ (resp. $\left.F C_{k, n+1}\right)$. Then

i) $N_{\pi_{w}^{n}, \pi_{w^{\prime}}^{n+1}}=N_{\pi_{w^{\prime}}^{n+1}, \pi_{w}^{n+2}}$.

ii) If $p$ is a minimal projection in $F C_{k, n}$ for $\pi_{w}^{n}$, then $p_{k} e_{n+1}=$ ${ }_{k} e_{n+1} p$ is a minimal projection in $F C_{k, n+2}$ for $\pi_{w}^{n+2}$.

Thus the vertices at the $n$-th step (i.e., representing matrix algebras of $\left.F C_{k, n}\right)$ are naturally identified with some of the vertices at the $(n+2)$-th step (i.e., some of the matrix algebras of $F C_{k, n+2}$ ). We now characterize the rest of the vertices at the $(n+2)$-th step, that is, those vertices indexed by $w$ so that $\operatorname{dim}\left(\pi_{w}^{n+2}\right)>0$ but $\pi_{w}^{n}=0$.

Lemma 12 ([BiJo1]). The following are equivalent when $w$ is a middle pattern for a diagram in $F C_{k, n} .\left(F C_{k, n}\right.$ need not be semisimple.)

1) $\pi_{w}^{n-2}=0$

2) $\operatorname{dim} \pi_{w}^{n}=1$.

3) $\pi_{w}^{n}\left({ }_{0} e_{j}\right)=0$ for all $j, 1 \leq j \leq n-1$.

4) $\pi_{w}^{n}\left({ }_{0} e_{j}\right)=0$ for some $j, 1 \leq j \leq n-1$.

5) No basis diagram for $\pi_{w}^{n}$ has $2(k+1)$ consecutive non-through strings.

Piecing the above two lemmas together we arrive at the following method for generating the Bratteli diagram for $F C_{k, n+1} \subset F C_{k, n+2}$, given the diagram for $F C_{k, n} \subset F C_{k, n+1}$ :

First reflect the Bratteli diagram for $F C_{k, n} \subset F C_{k+1, n}$ to get the "old stuff" in $F C_{k, n+2}$ (Lemma 11). Then add additional new vertices indexed by middle patterns that first occur in diagrams of $F C_{k, n+2}$. Each of these "new" vertices will be connected to only one vertex in $F C_{k, n+1}$ and that vertex will be one that was "new" in $F C_{k, n+1}$ (Lemma 12). Analyzing which middle patterns are "new" and which ones they are connected to is a straightforward application of Theorem 9.

Thus the first few levels of the Bratteli diagram for $F C_{2, n}$ is given by: 


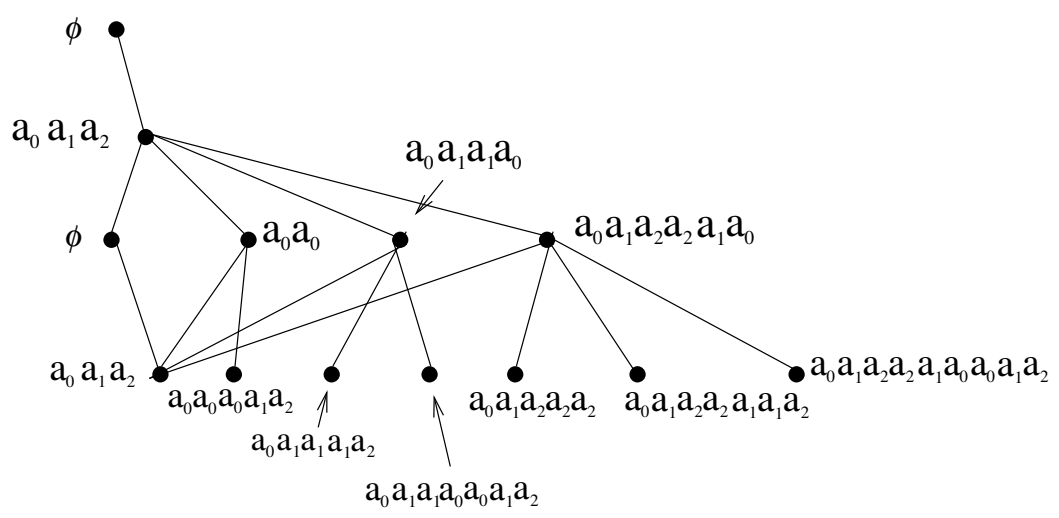

The above description actually tells us that writing down the full Bratteli diagram is redundant since the "old" stuff is just the reflection of a previous part of the Bratteli diagram. We can fully describe the Bratteli diagram by just describing the "new" stuff that appears at every level. This smaller graph is called the principal graph [GHJ], a term familiar to those who study subfactors. To clarify, the principal graph for the first few levels of $F C_{2, n}$ is given by:

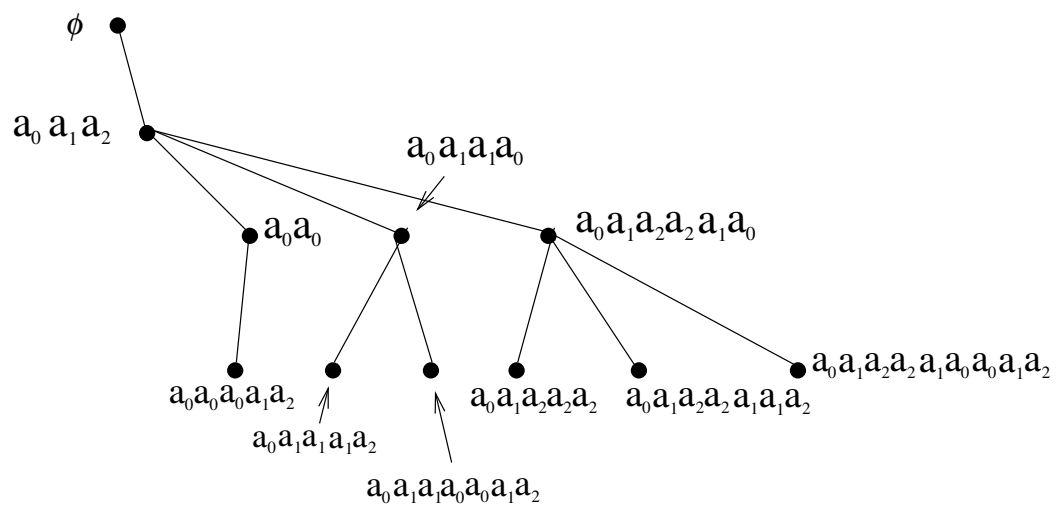

This brings us to a simple description of the Bratteli diagram for $F C_{k, n}$ :

Theorem 10. The principal graph $\Gamma_{k}$ for the inclusion

$$
F C_{k, 0} \subset F C_{k, 1} \subset F C_{k, 2} \subset \ldots
$$

is a tree inductively described as follows:

1) The 0-th level has one vertex labeled by 0.

2) Given a vertex on the $n$-th level labeled by $i$. Create $(i+1)$ new vertices on the $(n+1)$-th level with labels $k-i, k-i+1, \ldots k$.

3) Connect each of these new vertices to the given one on the $n$-th level.

To clarify, we give the principal graph $\Gamma_{k}$ for the first few levels: 


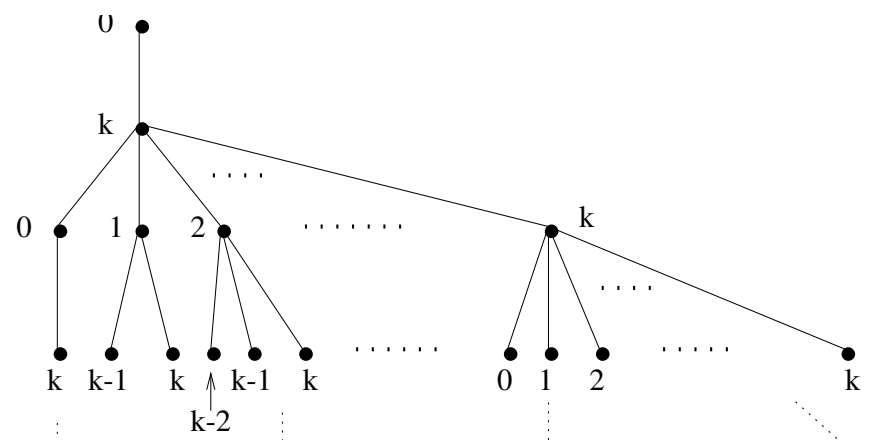

Proof. We use induction, using the characterization of new words $w$ as those for which no basis diagram for $\pi_{w}^{n}$ has $2(k+1)$ consecutive non-through strings (Lemma 12).

Let $\mathrm{P}(\mathrm{n})$ be the statement: A single diagram in $F C_{k, n}$ having the "new" middle pattern $w$ corresponding to a vertex on $\Gamma_{k}$ labeled by $i$ looks like:

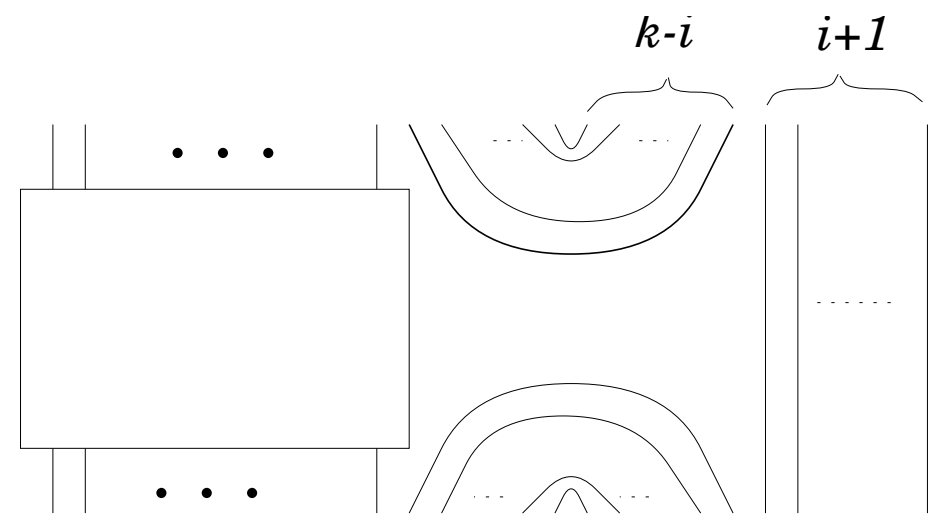

Clearly since $\operatorname{dim}\left(F C_{k, 1}\right)=1$, and the vertex corresponding to the word $a_{0} a_{1} \ldots a_{k}$ is labeled by $k$, we have $P(1)$ true.

Given $P(n)$ true consider $w$ corresponding to a vertex on the $n$th level of $\Gamma_{k}$ labeled by $i$. By the induction hypothesis the single diagram in $F C_{k, n}$ with middle pattern $w$ looks like the above figure. Without loss of generality, let us assume $n$ is even and so the rightmost label on the top of the diagrams is $a_{k}$. (The identical argument holds for $n$ odd if we replace $a_{k}$ with $a_{0}$.) Following the rule of Theorem 9 we can add or take away $a_{k}$ from $w$. If we add $a_{k}$, we will have to add $a_{k-1}$ and then $a_{k-2}$. . . to get just the new word $w a_{k} a_{k-1} \ldots a_{0}$. If we remove $a_{k}$ we will have the option to add or remove $a_{k-1}$. We continue in this way until we consider adding or removing $a_{k-i}$. If we remove $a_{k-i}$ then no matter what we do from then on we will have a middle pattern that has a diagram with $2(k+1)$ consecutive non-through strings and thus by Lemma 12 it will not correspond to a new 
middle pattern at the $(n+1)$-th level. Thus the only possibilities are the following $i+1$ middle patterns, indexed by $j, 0 \leq j \leq i$, none of which has $2(k+1)$ consecutive non-through strings.

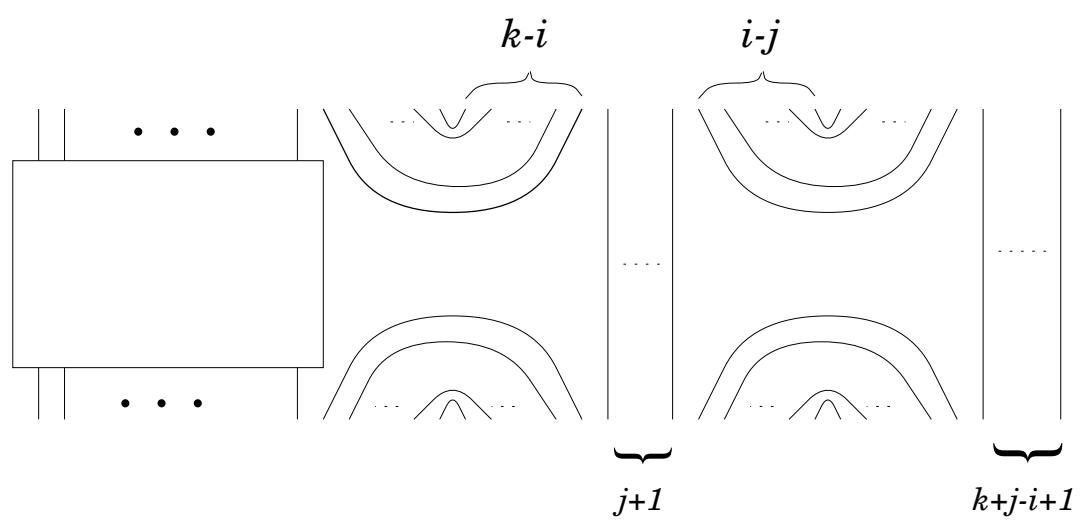

Labeling the vertex corresponding to the $j$-th middle pattern with $k-j+1$ gives us $P(n+1)$.

\section{Computation of $\left[\begin{array}{l}n \\ w\end{array}\right]$.}

To facilitate the ensuing discussion of paths on $\Gamma_{k}$ we introduce the following notation:

- Given a vertex $v$ of $\Gamma_{k}$, let $l(v)$ denote the label of $v$ (an integer between 0 and $k$ ).

- A path $P$ of length $n$ on $\Gamma_{k}$ will be written as $P=v_{0} v_{1} \ldots v_{n}$ where the $v_{i}$ are the successive vertices on $\Gamma_{k}$ encountered when following the path $P$.

- Denote by $f(w)$, the smallest $n$ such that $w$ appears as a middle pattern in $F C_{k, n}$.

- Each middle pattern $w$ corresponds to a vertex of the principal graph described above. There is a unique shortest path $P_{w}=v_{0} v_{1} \ldots v_{f(w)}$ along the principal graph $\Gamma_{k}$ (since the principal graph is a tree) from the root to the vertex indexed by $w$. Define $L(w)$ be the sequence $0, k, l\left(v_{2}\right), l\left(v_{3}\right) \ldots l\left(v_{f(w)}\right)$.

Corollary 10.1. Write $w=a_{s(1)} a_{s(2)} \ldots a_{s(d)}$ where the $s(i) \in[0, k]$. Let $S=\{i: s(i)=s(i+1)\}$. Then $f(w)=|S|+1$. Let $i_{1}<i_{2}<\cdots<i_{|S|}$ be the elements of $S$. Set $P_{w}=v_{0} v_{1} \ldots v_{f(w)}$ as above, the shortest path from the root to the vertex labeled by $w$ corresponding to the summand in $F C_{k, f(w)}$ 
indexed by $w$. Then we have:

$$
l\left(v_{j}\right)= \begin{cases}0 & \text { for } j=0, \\ k & \text { for } j=1, \\ s\left(i_{j-1}\right) & \text { for } j \text { even, } \\ k-s\left(i_{j-1}\right) & \text { for } j \text { odd. }\end{cases}
$$

Proof. This follows from the argument given in Theorem 10.

We have given an inductive description of the Bratteli diagram. In this section we get an explicit formula for $\left[\begin{array}{l}n \\ w\end{array}\right]$.

Consider all paths of length $2 n$ on $\Gamma_{k}$ with the following two properties:

1) The paths begin and end at the same vertex $v$.

2) No vertex of the path is closer to the root than $v$.

We note that the structure of $\Gamma_{k}$ guarantees that the same number of such paths will exist from two vertices $v$ and $w$ if $l(v)=l(w)$. This allows us to define $c_{i}^{n}$ to be the number of such paths of length $2 n$ starting and ending at a vertex $v$ with $l(v)=i$. Let $C_{i}(x)=\sum_{n=0}^{\infty} c_{i}^{n} x^{n}$, with the convention $c_{i}^{0}=1$.

Lemma 13. With the above notation we have

$$
C_{i}(x)=T^{i+1}(x)
$$

where $T$ is the generating function encountered previously (Section 5) satisfying:

$$
T=x T^{k+2}+1
$$

Proof. Let $P=v_{0} v_{1} \ldots v_{2 n}$ be an arbitrary path counted by $c_{i}^{n}$. (This means that $v_{0}=v_{2 n}$ with $l\left(v_{0}\right)=i$ and no $v_{i}$ closer to the root than $v_{0}$.) Let $i_{0}=\min \left\{i: i \geq 1\right.$ and $\left.v_{i}=v_{0}\right\}$. Write $P=P_{1} P_{2}$ with $P_{1}=v_{0} v_{1} \ldots v_{i_{0}}$ and $P_{2}=v_{i_{0}+1} v_{i_{0}+2} \ldots v_{2 n}$. The nature of $P$ and $\Gamma_{k}$ ensures that $v_{1}=v_{i_{0}-1}$ and thus the various possibilities for $P_{1}$ are counted by $\frac{\frac{i_{0}-2}{2}}{c_{l\left(v_{1}\right)}}$. On the other hand, the various possibilities for $P_{2}$ are counted by $c_{i}^{n-\frac{i_{0}}{2}}$. Since $l\left(v_{1}\right)$ can be any of $k-i, k-i+1 \ldots k$, we have the recursion:

$$
c_{i}^{n}=\sum_{j=k-i}^{k} \sum_{m=0}^{n-1} c_{j}^{m} c_{i}^{n-m-1}
$$

along with $c_{i}^{0}=1$.

This becomes:

$$
C_{i}=1+\sum_{j=k-i}^{k} x C_{j} C_{i} .
$$


Setting $D_{k-i}=x C_{i}$, and setting $i^{\prime}=k-i,(11)$ becomes

$$
D_{i^{\prime}}=x+\sum_{j^{\prime}=0}^{k-i^{\prime}} D_{j^{\prime}} D_{i^{\prime}}
$$

the same recusion relation as $A_{i}(x)$ in Equation (3), and thus all the results of Section 5 apply. Since $x C_{i}=D_{k-i}=A_{k-i}=L_{k-i-1}-L_{k-i}$, it follows from (9) that

$$
L_{k-i-1}-L_{k-i}=T^{-(k-i)}-T^{-(k-i+1)}=T^{i-k-1}(T-1)=x T^{i+1},
$$

where the last equality comes from substituting the known recursion relation for $T$ given in Equation (1).

Recall that given a middle pattern $w$, Corollary 10.1 allows us to calculate $f(w)$. In addition, it allows us to calculate $l\left(v_{j}\right)$ for $P=v_{0} v_{1} \ldots v_{f(w)}$ the unique shortest path from the root of $\Gamma_{k}$ to the vertex corresponding to the summand labeled by middle pattern $w$. With this notation we have:

\section{Theorem 11.}

$$
\left[\begin{array}{l}
n \\
w
\end{array}\right]=\text { the coefficient of } x^{n-f(w)} \text { in } T^{\sum_{i=0}^{f(w)}\left(l\left(v_{i}\right)+1\right)} .
$$

Setting $r=\sum_{i=0}^{f(w)}\left(l\left(v_{i}\right)+1\right)$,

$$
\left[\begin{array}{l}
n \\
w
\end{array}\right]=\left(\begin{array}{c}
(k+2)(n-f(w))+r \\
n-f(w)
\end{array}\right) \frac{r}{(k+2)(n-f(w))+r} .
$$

Proof. An arbitrary path of length $n$ from the root of $\Gamma_{k}$ to the $v_{f(w)}$ can be described as composition of paths as follows:

(a path counted by $\left.c_{l\left(v_{0}\right)}^{n_{0}}\right)$ (a path counted by $\left.c_{l\left(v_{1}\right)}^{n_{1}}\right) \cdots$

$$
\text { (a path counted by } \left.c_{l\left(v_{f(w)}\right)}^{n_{f(w)}}\right)
$$

with

$$
n_{0}+n_{1}+\ldots n_{f(w)}=n-f(w) .
$$

Thus we have

$$
\left[\begin{array}{l}
n \\
w
\end{array}\right] \text { is the coefficient of } x^{n-f(w)} \text { in } \prod_{i=0}^{f(w)} T^{l\left(v_{i}\right)+1} .
$$

Finally $[\mathbf{G K P}]$ gives the closed form expression for $T$ to an arbitrary power. 


\section{The Trace.}

The Fuss-Catalan algebras have a natural trace as described in the following definition:

Definition 18 ([BiJo1]). The Markov trace $\operatorname{tr}$ on $F C_{k, n}\left(\alpha_{0}, \alpha_{1}, \ldots \alpha_{k}\right)$ is defined on a diagram $D \in \mathcal{B}(k, n)$ as follows: tie the top of $\mathrm{D}$ to the bottom (the "types" $a_{i}$ will match up) and let $n_{i}$ be the number of loops of "type" $a_{i}$. Then set

$$
\operatorname{tr}(D)=\prod_{i=1}^{k} \alpha_{i}^{n_{i}-n} .
$$

Extend it by linearity to all of $F C_{k, n}$.

It is worth noting that this trace is consistent with the natural inclusion of $F C_{k, n} \subset F C_{k, n+1}$ given by adding $k+1$ vertical lines to the end of diagrams in $F C_{k, n}$.

Up to normalization, there is just one trace on a full matrix algebra. Thus to describe the trace on a direct sum of matrix algebras one need only give the relative weights on each of the summands. The standard way to do this is to give the value of the trace on a minimal projection of each summand. Section 3.3 of [BiJo1] explicitly describes the minimal projections (and the corresponding trace values) for $F C_{1, n}$. The general situation follows virtually the identical argument. For this reason we will merely state the results for $F C_{k, n}$ without proving most of the details.

We begin by recalling the situation for the Temperley-Lieb algebra (i.e., $\left.F C_{0, n}\right)[\mathbf{G H J}]$. Here an inductively defined series of polynomials is given by $P_{0}(\tau)=0, P_{1}(\tau)=1$ and $P_{l+1}(\tau)=P_{l}(\tau)-\tau P_{l-1}(\tau)$. Then if $1 /\left(\alpha_{0}\right)^{2}$ is not a root of $P_{l}(\tau)$ for $l \leq n+1$ the minimal projection in $T L_{n}\left(\alpha_{0}\right)$ corresponding to the summand with middle pattern $\left(a_{0}\right)^{n}$ has trace of $P_{n}\left(\left(\frac{1}{\alpha_{0}}\right)^{2}\right)$. We denote this projection by $f_{n}\left(a_{0}\right)$ and draw it as

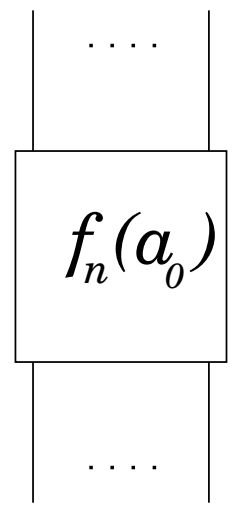

We note that $f_{n}\left(a_{0}\right)$ is a linear combination of elements of $\mathcal{B}(0, n)$. 
Theorem 12. Suppose the $F C_{k, n}\left(a_{0}, a_{1}, \ldots \alpha_{k}\right)$ are semisimple and none of the $\left(\alpha_{i}\right)^{-2}$ are zeros of $P_{l}(\tau), 1 \leq l \leq n$. Let $w$ be a middle pattern appearing for the first time inside $F C_{k, n}$. Write $w=a_{d_{1}}^{n_{1}} a_{d_{2}}^{n_{2}} \ldots a_{d_{m}}^{n_{m}}$ where $d_{i} \neq d_{i}+1$. If we denote by $D_{\text {in }}$ and $D_{\text {fin }}$ the initial and final parts of the only element of $\mathcal{B}(k, n)$ with middle pattern $w$, then the following diagram

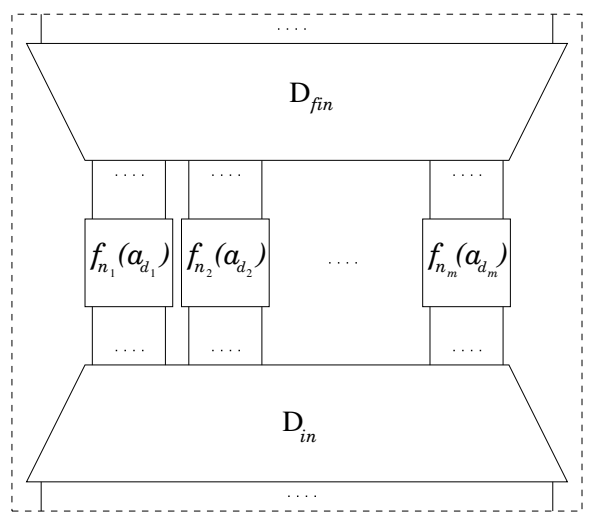

multiplied by the scalar

$$
\left(\frac{\alpha_{d_{1}}^{n_{1}} \ldots \alpha_{d_{m}}^{n_{m}}}{\left(\alpha_{0} \ldots \alpha_{k}\right)^{n}}\right)^{\frac{1}{2}}
$$

is the minimal idempotent corresponding to the simple summand indexed by $w$.

It is thus straightforward to see that the trace of the projection given in the above theorem is:

$$
\left(\frac{\alpha_{d_{0}}^{n_{0}} \ldots \alpha_{d_{m}}^{n_{m}}}{\left(\alpha_{0} \ldots \alpha_{k}\right)^{2 n}}\right)^{-\frac{1}{2}} \prod_{i=1}^{m} P_{n}\left(\left(\alpha_{i}\right)^{-2}\right) .
$$

Furthermore, by Lemma 11, the trace of a minimal projection corresponding to a summand $w$ inside $F C_{n^{\prime}, k}$ is the trace of the minimal projection corresponding to $w$ when it first appeared, say in $F C_{k, n}$, multiplied by $\left(\alpha_{0} \ldots \alpha_{k}\right)^{2\left(n-n^{\prime}\right)}$.

\section{Chains of Subfactors.}

We now come to the connection to subfactors that motivates the interest in the algebras $F C_{k, n}$. We consider the following situation: a pair of subfactors $N \subset M,[M: N]<\infty$, with a chain of intermediate subfactors

$$
N \subset P^{1} \subset P^{2} \subset \ldots P^{k} \subset M .
$$

As mentioned in the introduction, much of the information about the subfactor (and in some cases all of it [Po2]) is contained in what is called the (opposite) standard invariant [Po1]: 
In general, given a $\mathrm{II}_{1}$ subfactor $N \subset M$, we can perform the basic construction to get a projection $e_{1}$ and a new subfactor $M_{1}=\left\{M, e_{1}\right\}^{\prime \prime}$. Iterating this process we get a series of projections $e_{1}, e_{2}, e_{3} \ldots$ and a chain of subfactors (see $[\mathbf{J o 1}]$ ):

$$
N \subset M \subset M_{1} \subset M_{2} \ldots
$$

Intersecting this chain with $N^{\prime}$ and $M^{\prime}$ respectively gives a double sequence of finite-dimensional algebras:

$$
\begin{aligned}
& N^{\prime} \cap N \quad \subset \quad N^{\prime} \cap M \quad \subset \quad N^{\prime} \cap M_{1} \subset \ldots \\
& \cup \quad \cup \\
& M^{\prime} \cap M \quad \subset \quad M^{\prime} \cap M_{1} \subset \ldots
\end{aligned}
$$

known as the standard invariant. Now in any subfactor we have

$$
\begin{aligned}
& \left\{e_{1}, e_{2} \ldots e_{n}\right\} \subset N^{\prime} \cap M_{n}, \\
& \left\{e_{2}, e_{3} \ldots e_{n}\right\} \subset M^{\prime} \cap M_{n} .
\end{aligned}
$$

Thus the description of the algebras $\left\{e_{1}, e_{2} \ldots e_{n}\right\}^{\prime \prime}$, the Temperley-Lieb algebras, gives an underlying basic structure for all standard invariants (see $[\mathbf{G H J}])$.

In the case of $N \subset M$ with a chain of $k$ intermediate subfactors, it is natural to ask what additional basic structure of the standard invariant is ensured by the presence of such a chain. It is this question that was answered for one intermediate subfactor in [BiJo1]. We now answer the question in the general case using the work that has been developed here.

The presence of $P^{1} \subset P^{2} \subset \ldots \subset P^{k}$ gives rise to the factors

$$
M \subset P_{1}^{k} \subset P_{1}^{k-1} \ldots P_{1}^{1} \subset M_{1},
$$

with $P_{1}^{a}=J\left(P^{a}\right)^{\prime} J$. Here, all the factors are acting on $L^{2}(M)$ and $J$ is the completion of the canonical anti-isomorphism given by $J(x)=x^{*}$ on $M$, which is dense in $L^{2}(M)$. Alternatively we have $P_{1}^{a}=\left\{M, e_{P^{a}}^{M}\right\}^{\prime \prime}$ where $e_{P a}^{M}$ is the projection from $L^{2}(M)$ to $L^{2}\left(P^{a}\right)$, i.e., $P_{1}^{a}$ is the result of the basic construction for the inclusion $P^{a} \subset M$.

Thus when we iterate the basic construction we get the tower:

$$
\begin{aligned}
N \subset P^{1} \subset P^{2} \subset \ldots \subset P^{k} \subset M \subset P_{1}^{k} \subset P_{1}^{k-1} \subset \ldots \\
\\
\quad \subset P_{1}^{1} \subset M_{1} \subset P_{2}^{1} \subset P_{2}^{2} \subset \ldots
\end{aligned}
$$

with

$$
P_{j}^{a}=\left\{M_{j-1}, e_{P_{j-1}^{a}}^{M_{j-1}}\right\}^{\prime \prime}
$$


To simplify notation we will let

$$
e_{a, j}= \begin{cases}e_{M_{j-2}}^{M_{j-1}} & \text { for } a=k \\ e_{P_{j-1}^{k-a}}^{M_{j-1}} & \text { for } j \text { odd, } a \neq k \\ e_{P_{j-1}^{a+1}}^{M_{j-1}} & \text { for } j \text { even, } a \neq k\end{cases}
$$

(The notation is similar to the notation for the generators for $A A_{k, n}$ above. It is hoped that the similarity will not cause confusion but rather give away the punch line, namely that the map from ${ }_{a} e_{j}$ to $e_{a, j}$ is generically an isomorphism.)

Thus for a subfactor with a chain of $k$ intermediate subfactors we have:

$$
\left\{1, e_{a, j}: 0 \leq a \leq k, 1 \leq j \leq n-1\right\} \subset N^{\prime} \cap M_{n},
$$

and

$$
\left\{1, e_{a, j}: 0 \leq a \leq k, 2 \leq j \leq n-1\right\} \subset M^{\prime} \cap M_{n} .
$$

It is the structure of the algebra generated by the set $\left\{1, e_{a, j}: 0 \leq a \leq\right.$ $k, 1 \leq j \leq n-1\}$ that we seek.

Definition 19. Let

$$
\begin{aligned}
& \alpha_{0}=\left[P^{1}: N\right]^{\frac{1}{2}} \\
& \alpha_{a}=\left[P^{a+1}: P^{a}\right]^{\frac{1}{2}} \quad \text { for } 1 \leq a \leq k-1 \\
& \alpha_{k}=\left[M: P^{k}\right]^{\frac{1}{2}}
\end{aligned}
$$

where $[M: N]$ is the index of the inclusion of $N \subset M$ (see [Jo1] for definition of index).

Let $I A_{k, n}\left(\alpha_{0}, \alpha_{1}, \ldots \alpha_{k}\right)$ be the algebra generated by the set $\left\{1, e_{a, j}: 0 \leq\right.$ $a \leq k, 1 \leq j \leq n-1\}$.

Lemma 14. Given $I A_{k, n}$ as above we have:

1) $e_{a, i} e_{b, i}=e_{\max (a, b), i}$,

2) $e_{a, i} e_{b, j}=e_{b, j} e_{a, i} \quad$ for $|i-j| \geq 2$ or, $|i-j|=1$ and $a+b<k$

3) $e_{a, i} e_{b, i \pm 1} e_{a, i}=c(a, b) e_{a, i} e_{k-a-1, i \pm 1} \quad$ for $a+b \geq k\left(e_{-1, i \pm 1}=1\right)$ with $c(a, b)$ as in Definition 1:

$$
\begin{aligned}
c(a, b)=\frac{k-a-1}{{ }_{a} c_{i+1} c_{i} b-1} c_{i+1} c_{i} \\
\text { where } \quad{ }_{a} c_{i}=\left\{\begin{array}{ll}
\alpha_{0} \cdots \alpha_{a} & \text { for } i \text { even } \\
\alpha_{k} \cdots \alpha_{k-a} & \text { for } i \text { odd. }
\end{array} \quad \text { and }{ }_{-1} c_{i}=1 .\right.
\end{aligned}
$$


Proof. We assume $i$ even; identical arguments hold for $i$ odd. Relation 1 follows from the fact that $P_{i-1}^{\max (a, b)+1} \subset P_{i-1}^{a+1}, P_{i-1}^{b+1}$. Relation 2 follows from $\left\{e_{a, i}\right\}^{\prime} \cap M_{i-1}=P_{i-1}^{a+1}$. Relation 3 follows from [BiJo1, Prop. 5.1].

Lemma 15. Let $\operatorname{tr}$ be the trace on $M_{n}$ restricted to $I A_{k, n}$. Then $\operatorname{tr}$ is a Markov trace, that is,

$$
\begin{gathered}
\operatorname{tr}(1)=1, \\
\operatorname{tr}\left(x e_{a, n}\right)=\frac{1}{\left({ }_{a} c_{n}\right)^{2}} \operatorname{tr}(x) \text { for all } x \in I A_{k, n} .
\end{gathered}
$$

Proof. The proof follows from the fact that the conditional expectations are trace preserving:

$$
\operatorname{tr}\left(x e_{a, n}\right)=\operatorname{tr}\left(E_{M_{n-1}}^{M_{n}}\left(x e_{a, n}\right)\right)=\operatorname{tr}\left(x E_{M_{n-1}}^{M_{n}}\left(e_{a, n}\right)\right)=\frac{1}{\left({ }_{a} c_{n}\right)^{2}} \operatorname{tr}(x) .
$$

Theorem 13. There is a surjective, trace-preserving, unital *-homomorphism

$$
\iota: F C_{k, n}\left(\alpha_{0} \ldots \alpha_{k}\right) \longrightarrow I A_{k, n}\left(\alpha_{0} \ldots \alpha_{k}\right)
$$

given by

$$
\begin{gathered}
\iota(1)=1, \\
\iota\left({ }_{a} e_{i}\right)=e_{a, i}, \quad 0 \leq a \leq k, \quad 1 \leq i \leq n-1 .
\end{gathered}
$$

Proof. By definition of $I A_{k, n}, \iota$ is clearly onto. That $\iota$ is a ${ }^{*}$-homomorphism is established by Lemma 14 . We now show $\iota$ is trace-preserving by showing that the properties:

$$
\begin{gathered}
\operatorname{tr}(1)=1, \\
\operatorname{tr}\left(x_{a} e_{n}\right)=\frac{1}{\left({ }_{a} c_{n}\right)^{2}} \operatorname{tr}(x) \text { for } x \in A A_{k, n},
\end{gathered}
$$

completely determine the value of the trace on $\cup_{n=0}^{\infty} F C_{k, n}$. The form of words in the basis $S_{k, n}$ of $A A_{k, n} \cong F C_{k, n}$ indicates that a word $v \in F C_{k, n+1}$ can be written as $v=x_{a} e_{n} y$ with $x$ and $y$ in $F C_{k, n}$. Since the trace property gives us that

$$
\operatorname{tr}(v)=\operatorname{tr}\left(x_{a} e_{n} y\right)=\operatorname{tr}\left(y x_{a} e_{n}\right)=\frac{1}{\left({ }_{a} c_{n}\right)^{2}} \operatorname{tr}(y x),
$$

we have by induction that the above properties determine the value of the trace everywhere. Since the trace on $F C_{k, n}$ clearly satisfies the above two properties, $\iota$ is trace preserving.

In many cases $\iota$ is an isomorphism:

Theorem 14. Suppose $\alpha_{i} \geq 2$ for $0 \leq i \leq k$. Then the homomorphism $\iota: F C_{k, n} \longrightarrow I A_{k, n}$ in Theorem 13 is an isomorphism. Thus $F C_{k, n}$ is semisimple. 
Proof. Let $F C_{0}(\alpha)=\cup_{i=0}^{\infty} F C_{0, i}(\alpha)$. (The $F C_{0, i}(\alpha)$ are generically the Temperley-Lieb algebras.) Consider the trace TR on

$$
F C_{0}\left(\alpha_{0}\right) \otimes F C_{0}\left(\alpha_{1}\right) \otimes \cdots \otimes F C_{0}\left(\alpha_{k}\right)
$$

given by the tensor product of the traces on $F C_{0}\left(\alpha_{i}\right)$. It is known that the trace on the Temperley-Lieb algebra $T L\left(\alpha_{i}\right)$ is faithful for $\alpha_{i} \geq 2[\mathbf{G H J}]$. Since the tensor product of faithful traces is faithful [Di], TR is faithful. We can think of $F C_{k, n} \subset F C_{0, n}\left(\alpha_{0}\right) \otimes F C_{0, n}\left(\alpha_{1}\right) \otimes \cdots \otimes F C_{0, n}\left(\alpha_{k}\right)$ in the following way: view a diagram in $F C_{k, n}$ as an overlay of $k+1$ diagrams, each diagram consisting of only strings labeled by $a_{i}, 0 \leq i \leq k$. Each of these is an element of $F C_{0, n}\left(\alpha_{i}\right)$ and since multiplication in $F C_{k, n}$ does not combine strings with different labels, the multiplicative structure of $F C_{k, n}$ agrees with that of $F C_{0}\left(\alpha_{0}\right) \otimes F C_{0}\left(\alpha_{1}\right) \otimes \cdots \otimes F C_{0}\left(\alpha_{k}\right)$. It is clear that $\left.T R\right|_{F C_{k, n}}$ is just the usual trace on $F C_{k, n}$, and thus $\operatorname{tr}$ on $F C_{k, n}$ is faithful. Since $\iota$ is trace preserving for a faithful trace, $\operatorname{ker}(\iota)=\{0\}$ and $\iota$ an isomorphism.

The important condition for the above isomorphism was the faithfulness of the trace. To determine the situation for the non-generic case we merely have to examine to what extent the trace on $F C_{k, n} \subset F C_{0, n}\left(\alpha_{0}\right) \otimes F C_{0, n}\left(\alpha_{1}\right) \otimes$ $\cdots \otimes F C_{0, n}\left(\alpha_{k}\right)$ is not faithful.

Recall that $\Gamma_{k}$ is the principal graph for the inclusion $F C_{k, 0} \subset F C_{k, 1} \subset$ $F C_{k, 2} \ldots$ Each node of $\Gamma_{k}$ is labeled by a "new" middle pattern $w$ in the alphabet $\left\{a_{0}, a_{1}, \ldots, a_{k}\right\}$. Let $v=\left(v_{0}, v_{1}, \ldots, v_{n}\right) \in\{\mathbf{N} \cup \infty\}^{k}$. Define

$W_{v}=\left\{w: w\right.$ does not have a string of consecutive $a_{i}$ 's longer than $\left.v_{i}\right\}$.

Define $\Gamma_{k, v}$ to be the subgraph of $\Gamma_{k}$ consisting only of nodes labeled by $w \in W_{v}$.

Theorem 15. Given

$$
\left(\alpha_{0}, \alpha_{1}, \ldots, \alpha_{k}\right), \quad \alpha_{i} \in\left\{2 \cos \frac{\pi}{m}: m \geq 2\right\} \cup[2, \infty),
$$

set

$$
v_{i}= \begin{cases}m & \text { if } \alpha_{i}=2 \cos \frac{\pi}{m} \\ \infty & \text { if } \alpha_{i} \in[2, \infty)\end{cases}
$$

Then $\Gamma_{k, v}$ is the principal graph for the tower

$$
\mathbb{C} \subset I A_{k, 1} \subset I A_{k, 2} \subset \ldots
$$

Proof. Define the ideal $I_{n}^{j}$ to be the ideal generated by $\left\{x^{*} x: x \in F_{0, n}\left(\alpha_{j}\right)\right.$ and $\left.\operatorname{tr}\left(x^{*} x\right)=0\right\}$. Again considering $F C_{k, n} \subset F C_{0, n}\left(\alpha_{0}\right) \otimes F C_{0, n}\left(\alpha_{1}\right) \otimes$ $\cdots \otimes F C_{0, n}\left(\alpha_{k}\right)$ as in the proof of Theorem 14, we have $\iota$ factors through the algebra:

$$
\begin{gathered}
B=F C_{0, n}\left(\alpha_{0}\right) / I_{n}^{0} \otimes F C_{0, n}\left(\alpha_{1}\right) / I_{n}^{1} \otimes \cdots \otimes F C_{0, n}\left(\alpha_{k}\right) I_{n}^{k}: \\
\iota: F C_{k, n} \stackrel{\iota_{1}}{\longrightarrow} B \stackrel{\iota_{2}}{\longrightarrow} I A_{k, n} .
\end{gathered}
$$


Because the tensor product of faithful traces is faithful, we have that the trace on $B$ is faithful. Therefore since $\iota_{2}$ is trace preserving we have $\iota_{2}$ an isomorphism. Thus for $\iota: F C_{k, n} \longrightarrow I A_{k, n}, \operatorname{ker}(\iota)$ is the intersection of $F C_{k, n}$ and the ideal generated by

$$
\left\{I_{n}^{0} \otimes 1 \otimes 1 \otimes \cdots \otimes 1 \cup 1 \otimes I_{n}^{1} \otimes 1 \otimes \cdots \otimes 1 \cup \cdots \cup 1 \otimes 1 \otimes \cdots \otimes I_{n}^{k}\right\} .
$$

Let $p$ be a diagram that is a projection in $F C_{k, n}$ with middle pattern $w$. It follows that $p \in \operatorname{ker}(\iota) \bmod F C_{k, n} k_{n-1} F C_{k, n}$ if and only if $w \notin W$. The result now follows by applying this fact at every node of the Bratteli diagram $\Gamma_{k}$.

This completes the description of the underlying structure of the standard invariant given by the presence of a chain of intermediate subfactors. When considering a subfactor $N \subset M$, we can ask the following questions. Does $N \subset M$ contain chains of intermediate subfactors? If so, how can we use the added structural information obtained in this work to help determine the entire structure of the standard invariant? The answer to the first question is provided in [Bi1]. Work in the direction of the second question appears in [BiJo3], where dimensional criterea are given for the standard invariant to be exactly $F C_{1, n}$, and in [La], where for certain examples, the standard invariants of $N \subset P$ and $P \subset M$ are used to determine the standard invariant of $N \subset M$.

\section{References}

[Bh] B. Bhattacharyya, Krishnan-Sunder subfactors and a new countable family of subfactors related to trees, $\mathrm{PhD}$ Thesis, UC Berkeley, 1998.

[Bi1] D. Bisch, A note on intermediate subfactors, Pac. J. Math., 163(2) (1994), 201216.

[Bi2] Bimodules, higher relative commutants and the fusion algebra associated to a subfactor, The Fields Institute for Research in Mathematical Sciences Communications Series, 13 (1997), 13-63.

[BiJo1] D. Bisch and V.F.R. Jones, Algebras associated to intermediate subfactors, Invent. Math., 128(1) (1997), 89-157.

[BiJo2] _ A note on free composition of subfactors, Lecture Notes in Pure and Appl. Math., 284 (1997), 339-361, Dekker, New York.

[BiJo3] _ Singly generated planar algebras of small dimension, Duke Math. J., 101(1) (2000), 41-75.

[DFr] P. Di Francesco, New integrable lattice models from Fuss-Catalan algebras, Nuclear Phys., B 532(3) (1998), 609-634.

[Di] J. Dixmier, Von Neumann Algebras, North-Holland Mathematical Library, 27, North-Holland Publishing Co., Amsterdam-New York, 1981.

[GHJ] F. Goodman, P. de la Harpe and V.F.R. Jones, Coxeter Graphs and Towers of Algebras, Springer Verlag, MSRI publications, 1989. 
[GKP] R. Graham, D. Knuth and O. Patashnik, Concrete Mathematics, Addison-Wesley Publishing Company, 2nd edition, 1994.

[He] P. Hersh, A bijective proof of the dimension of the Fuss-Catalan algebras, informal note, www.math.washington.edu/ hersh/papers.html.

[Jo1] V.F.R. Jones, Index for subfactors, Invent. Math., 72 (1983), 1-25.

[JS] V.F.R. Jones and V.S. Sunder, Introduction to Subfactors, London Mathematical Society Lecture Note Series, 234 (1997).

[Ka] L.H. Kauffman, State models and the Jones polynomial, Topology, 26(3) (1987), 395-407.

[La] Z. Landau, Intermediate Subfactors, PhD Thesis, UC Berkeley, 1998.

[PiPo] M. Pimsner and S. Popa, Entropy and Index for Subfactors, Annales Scientifiques de L'Ecole Normale Superieure, 4 serie, t. 19, 1986.

[Po1] S. Popa, Classification of amenable subfactors of type II, Acta Math., 172 (1994), 352-445.

[Po2] _ Classification of Subfactors and their Endomorphisms, CBMS Regional conference series in mathematics, 86 (1995).

[Po3] - An axiomatization of the lattice of higher relative commutants of a subfactor, Invent. Math., 120(3) (1995), 427-445.

[St] R. Stanley, Enumerative Combinatorics, Vol. 2, Cambridge University Press, 1999.

Received May 1, 1999 and revised April 27, 2000.

Mathematical Science Research Institute

1000 Centennial Drive, \#5070

BERKELEY, CA 94720-5070

E-mail address: landau@math.berkeley.edu 